\title{
Upconversion nanoparticle-mediated photodynamic therapy induces THP-I macrophage apoptosis via ROS bursts and activation of the mitochondrial
} caspase pathway

\author{
This article was published in the following Dove Press journal: \\ International Journal of Nanomedicine \\ 22 May 2015 \\ Number of times this article has been viewed
}

\begin{abstract}
Xing Zhu, ',* Hao Wang, ${ }^{2, *}$ Longbin Zheng,' Zhaoyu Zhong,' Xuesong Li,' Jing Zhao, ${ }^{3}$ Jiayuan Kou, ' Yueqing Jiang,' Xiufeng Zheng,' Zhongni Liu,' Hongxia Li,' Wenwu Cao, ${ }^{4,5}$ Ye Tian, ${ }^{1,6}$ You Wang, ${ }^{2}$ Liming Yang'

'Department of Pathophysiology, Harbin Medical University, Harbin, People's Republic of China; ${ }^{2}$ Materials Physics and Chemistry Department, Harbin Institute of Technology, Harbin, People's Republic of China; ${ }^{3}$ Blood Transfusion Department, Jining No I People's Hospital, Jining, People's Republic of China;

${ }^{4}$ Laboratory of Sono- and Phototheranostic Technologies, Harbin Institute of Technology, Harbin, People's Republic of China; ${ }^{5}$ Materials Research Institute, The Pennsylvania State University, University Park, PA, USA; ${ }^{6}$ Division of Cardiology, The First Affiliated Hospital, Harbin Medical University, Harbin, People's Republic of China

*These authors contributed equally to this work
\end{abstract}

Correspondence: Liming Yang

Department of Pathophysiology, Harbin Medical University, NO. 194 Xuefu Road, Nangang District, Harbin I5008I, People's Republic of China

Tel +8645 I 86674548

Fax +86 45I 86674548

Email limingyang@ems.hrbmu.edu.cn

You Wang

Materials Physics and Chemistry Department, Harbin Institute of Technology,

NO. 92 Xidazhi Street Nangang District,

Harbin I5000 I, People's Republic of China

Tel +86 45I 86403109

Fax +86 45I 864I 25I6

Email wangyouhit@।63.com
Abstract: Atherosclerosis (AS) is the most vital cardiovascular disease, which poses a great threat to human health. Macrophages play an important role in the progression of AS. Photodynamic therapy (PDT) has emerged as a useful therapeutic modality not only in the treatment of cancer but also in the treatment of AS. The purpose of this study was to determine the molecular mechanisms underlying the activity of PDT, using mesoporous-silica-coated upconversion fluorescent nanoparticles encapsulating chlorin e6 (UCNPs-Ce6) in the induction of apoptosis in THP-1 macrophages. Here, we investigated the ability of UCNPs-Ce6-mediated PDT to induce THP-1 macrophage apoptosis by facilitating the induction of reactive oxygen species (ROS) and regulation of mitochondrial permeability transition pore (MPTP) to depolarize mitochondrial membrane potential (MMP). Both Bax translocation and the release of cytochrome $\mathrm{C}$ were examined using immunofluorescence and Western blotting. Our results indicated that the levels of ROS were significantly increased in the PDT group, resulting in both MPTP opening and MMP depolarization, which led to apoptosis. In addition, immunofluorescence and Western blotting revealed that PDT induced both Bax translocation and the release of cytochrome $\mathrm{C}$, as well as upregulation of cleaved caspase-9, cleaved caspase-3, and cleaved poly(ADP-ribose) polymerase. Therefore, we demonstrated that UCNPs-Ce6-mediated PDT induces apoptosis in THP-1 macrophages via ROS bursts. The proapoptotic factor Bax subsequently translocates from the cytosol to the mitochondria, resulting in the MPTP opening and cytochrome $\mathrm{C}$ release. This study demonstrated the great potential of UCNPs-Ce6-mediated PDT in the treatment of AS.

Keywords: atherosclerosis, photodynamic therapy, fluorescent nanoparticles, reactive oxygen species, apoptosis, macrophages

\section{Introduction}

Plaques play an important role in the progression of atherosclerosis (AS), and myocardial infarction, stroke, and sudden cardiac death are each caused by the rupture of these plaques. ${ }^{1,2}$ As the vital cells in unstable atherosclerotic plaques, macrophages participate in a maladaptive, nonresolving inflammatory response that expands the subendothelial layer due to the accumulation of cells, lipid, and matrix, which subsequently lead to the formation of vulnerable plaques. ${ }^{2,3}$ Surgical treatment for AS, such as percutaneous coronary intervention, results in numerous complications. ${ }^{4}$ Therefore, the development of a new treatment for AS is desirable.

Photodynamic therapy (PDT) has emerged as one of the promising therapies in the treatment of cancers, and it is a type of noninvasive medical technology that consists of 
the following three key components: a photosensitizer, light, and molecular oxygen. ${ }^{5,6}$ PDT induces cell death via oxidative damage caused by cytotoxic reactive oxygen species (ROS: primarily singlet oxygen, ${ }^{1} \mathrm{O}_{2}$ ), which are produced as a result of the transfer of energy from an excited photosensitizer to adjacent oxygen molecules. ${ }^{7}$ PDT has also been used to treat cardiovascular pathologies, including AS and restenosis, ${ }^{8,9}$ and our group previously testified that 5-aminolevulinicacid-mediated PDT both decreases macrophage content and inhibits plaque progression in rabbit carotid artery AS models. ${ }^{10}$ However, a drawback of conventional therapy is the requirement of direct illumination of the tissue by either visible (VIS) or ultraviolet (UV) light to excite the photosensitizers, which have limited penetration depth due to both the absorption and scattering of light by biological tissues, thereby resulting in ineffective therapeutic effects. ${ }^{11,12}$ The use of near-infrared (NIR) light in PDT can afford greater penetration depths than that of VIS light because the absorbance for most biomolecules reaches a minimum in the NIR window (700-1,100 nm), ${ }^{13}$ and the usefulness of the upconversion nanoparticles (UCNPs) that can convert NIR light to VIS light for noninvasive imaging of deep tissues, drug delivery, and PDT has been shown. ${ }^{14-20}$ In this study, we loaded a commonly used second-generation photosensitizer, chlorin e6 (Ce6), onto silica-coated upconversion nanoparticles to form a supramolecular UCNPs-Ce6 complex used for NIR light-induced PDT of THP-1 macrophages, and we subsequently determined whether UCNPs-Ce6-mediated PDT induced apoptosis through the mitochondrial caspase pathway via ROS bursts, mitochondrial permeability transition pore (MPTP) opening, mitochondrial membrane potential (MMP) depolarization, and mitochondrial dysfunction in vitro.

\section{Materials and methods 980 nm PDT device}

The $980 \mathrm{~nm}$ PDT device was assembled by the Harbin Institute of Technology (Harbin, People's Republic of China). The laser diode of this device emits a single $980 \mathrm{~nm}$ excitation wavelength irradiation with a diameter of $2 \mathrm{~mm}$. The cells were placed $2 \mathrm{~cm}$ from the laser diode to receive PDT treatment, and the laser irradiation intensity was $2.0 \mathrm{~W}$. During this procedure, the temperature of the solution inside the Petri dishes increased less than $2^{\circ} \mathrm{C}$, as measured using a thermometer.

\section{Cell culture}

Human THP-1 monocytes were obtained from the American Type Culture Collection (ATCC, Manassas, VA, USA).
The cells were cultured using a split ratio of 1:10 in RPMI 1640 medium (HyClone, Logan, UT, USA) supplemented with $10 \%$ fetal bovine serum (FBS; HyClone), $20 \mu \mathrm{g} / \mathrm{mL}$ penicillin, and $20 \mu \mathrm{g} / \mathrm{mL}$ streptomycin (Sigma-Aldrich Co., St Louis, MO, USA). Culture was carried out at $37^{\circ} \mathrm{C}$ in a humidified incubator containing $5 \% \mathrm{CO}_{2}$, and the medium was refreshed every 2-3 days. For the experiment, the cells were seeded at a density of $1.0 \times 10^{5}$ cells $/ \mathrm{mL}$ and were differentiated into macrophages by adding $100 \mathrm{ng} / \mathrm{mL}$ phorbol12-myristate-13-acetate (EMD Biosciences Inc., La Jolla, CA, USA) for 72 hours.

\section{PDT treatment protocols}

THP-1 macrophages in the exponential phase were collected, differentiated, and randomly divided into the following four groups: 1) control, 2) UCNPs-Ce6 alone, 3) laser alone, and 4) PDT. For both the UCNPs-Ce6 group and the PDT group, the cells were incubated with $16 \mu \mathrm{g} / \mathrm{mL}$ UCNPs-Ce6 for a compound loading time of 4 hours in FBS-loaded RPMI 1640 medium. Instead of UCNPs-Ce6, an equivalent volume of medium was used in both the control and laser groups. The cells in both the laser and PDT groups were exposed to a $980 \mathrm{~nm}$ laser at an intensity of $2.0 \mathrm{~W}$ for 60 seconds. Following the treatments, the cells were carefully washed once in phosphate-buffered saline (PBS), cultured in fresh medium for an additional 6 hours and subjected to different analyses. For the inhibitory experiments, z-VAD-FMK, cyclosporin A (CsA), $N$-acetyl-L-cysteine (NAC), sodium azide $\left(\mathrm{NaN}_{3}\right)$, mannitol, superoxide dismutase (SOD), catalase (CAT), bongkrekic acid (BA), and 4,4'-diisothiocyanatostilbene2,2'-disulfonic acid disodium (DIDS) were added to the culture medium with UCNPs-Ce6 loading for 4 hours.

\section{Cell viability assay}

Cell viability was assessed by a CCK-8 assay (Beyotime Biotechnology, Inc., Beijing, People's Republic of China). Cells treated with phorbol-12-myristate-13-acetate $(100 \mathrm{ng} / \mathrm{mL})$ were seeded into 96-well plates and incubated for 72 hours at $37^{\circ} \mathrm{C}$ in the dark. The cells were then subjected to the following experimental treatments: 1$)$ the cells were incubated with different UCNPs-Ce6 concentrations $(0-64.0 \mu \mathrm{g} / \mathrm{mL})$ for 4 hours at $37^{\circ} \mathrm{C}$ in the dark; 2) the cells were incubated with $16 \mu \mathrm{g} / \mathrm{mL}$ UCNPs-Ce6 for $0-5$ hours; 3 ) the cells were exposed to laser for $0-150$ seconds; 4 ) the cells were incubated with different UCNPs-Ce 6 concentrations $(0-16 \mu \mathrm{g} / \mathrm{mL})$ for 4 hours and exposed to $980 \mathrm{~nm}$ laser for 60 seconds; 5) the cells were incubated with $16 \mu \mathrm{g} / \mathrm{mL}$ UCNPs-Ce 6 for 4 hours and exposed to $980 \mathrm{~nm}$ laser for $0-60$ seconds; 6) the cells 
were incubated with $16 \mu \mathrm{g} / \mathrm{mL}$ UCNPs-Ce6 for 4 hours and exposed to different capacities of $980 \mathrm{~nm}$ laser for 60 seconds; 7) the cells were incubated with $16 \mu \mathrm{g} / \mathrm{mL}$ UCNPs-Ce6 for $0-6$ hours, followed by 60 seconds of $980 \mathrm{~nm}$ laser; 8) the cells were incubated with $16 \mu \mathrm{g} / \mathrm{mL}$ UCNPs-Ce6 followed by $200 \mathrm{U} / \mathrm{mL}$ CAT (hydrogen peroxide scavenger), $100 \mu \mathrm{g} / \mathrm{mL}$ SOD (superoxide anion radical scavenger), $100 \mathrm{mM}$ mannitol (hydroxyl radical scavenger), $10 \mathrm{mM}$ $\mathrm{NaN}_{3}\left({ }^{1} \mathrm{O}_{2}\right.$ scavenger), or z-VAD-FMK (caspase inhibitor) at different concentrations $(5,10,20 \mu \mathrm{M})$ for 4 hours. After a 6-hour incubation following PDT, the medium was removed, and $100 \mu \mathrm{L}$ of medium containing CCK-8 (split ratio 10:1) without FBS was added to the cells, which were then incubated for 1 hour at $37^{\circ} \mathrm{C}$ in the dark. Absorption at $450 \mathrm{~nm}$ was subsequently measured on a microplate reader (BioTek Elx800, BioTek, Winooski, VT, USA). The data represent the averages for six wells for each treatment, and each experiment was repeated three times individually.

\section{Hoechst 33258 and propidium iodide assays}

Six hours after UCNPs-Ce6-PDT treatment, the cells were washed with PBS and stained with Hoechst 33258 and propidium iodide (PI), according to the manufacturer's instructions. Briefly, the macrophages were incubated with $10 \mu \mathrm{g} / \mathrm{mL}$ PI for 10 minutes at $37^{\circ} \mathrm{C}$ in the dark, and then incubated with $5 \mu \mathrm{g} / \mathrm{mL}$ Hoechst 33258 for 10 minutes. The cells were washed twice with PBS and then examined under a fluorescence microscope (Olympus IX81, Olympus Corporation, Tokyo, Japan) using a filter with an excitation wavelength of 330-385 $\mathrm{nm}$ and an emission wavelength of 420-480 nm. The necrotic cells were stained with red fluorescence, while apoptotic cells were stained with blue fluorescence. The fractions of apoptotic or necrotic cells were determined using five random microscopic images with at least 1,000 cells/group. Experiments were repeated three times independently.

\section{TUNEL staining}

Six hours after the UCNPs-Ce6-PDT treatment, the air-dried cells were fixed with $4 \%$ paraformaldehyde for 1 hour at $15^{\circ} \mathrm{C}-25^{\circ} \mathrm{C}$. The fixed cells were washed twice with PBS prior to incubation with $3 \% \mathrm{H}_{2} \mathrm{O}_{2}$ in methanol for 10 minutes at $15^{\circ} \mathrm{C}-25^{\circ} \mathrm{C}$. The cells were then incubated in $0.1 \%$ Triton $\mathrm{X}-100$ in $0.1 \%$ sodium citrate for 2 minutes on ice after being washed with PBS twice. The prepared cells were then labeled with a TUNEL reaction mixture for 60 minutes at $37^{\circ} \mathrm{C}$ in a humidified atmosphere in the dark, according to the manufacturer's instructions for the In Situ Cell Death Detection Kit, POD (Catalog No 11684817910, Roche Diagnostics, Mannheim, Germany). The cells were stained with $1.0 \mu \mathrm{g} / \mathrm{mL}$ DAPI (4',6-diamidino-2-phenylindole) for 5 minutes to visualize the nuclei and were then washed twice with PBS and examined under a fluorescence microscope (Olympus IX81, Olympus Corporation) using a filter.

\section{Detection of singlet oxygen generation}

Singlet oxygen $\left({ }^{1} \mathrm{O}_{2}\right)$ generation following PDT treatment was measured by chemical oxidation of 9,10-anthracenediylbis (methylene) dimalonic acid (ABDA) in the aqueous solution. In a typical experiment, $16 \mu \mathrm{g} / \mathrm{mL}$ UCNPs-Ce6 was suspended in $1 \mathrm{~mL}$ of water containing $10 \mu \mathrm{M}$ ABDA dye. For a control sample used to verify the involvement of ${ }^{1} \mathrm{O}_{2}$ in the fluorescence decay of ABDA, $10 \mathrm{mM} \mathrm{NaN}_{3}$ (a scavenger of ${ }^{1} \mathrm{O}_{2}$ ) was also included in the mixture. The mixture was then placed in a cuvette and the solution was irradiated with $980 \mathrm{~nm}$ laser at $2.0 \mathrm{~W}$ for 10 second time intervals, beginning from time $(t)=0$ and continuing for 5 minutes, and the emission fluorescence of ABDA (upon excitation at $380 \mathrm{~nm}$ ) was measured between intervals using a fluorescence spectrophotometer (Varian Australia Pty, Ltd, Melbourne, VIC, Australia).

\section{Measurement of intracellular ROS}

Intracellular ROS content was determined by measuring the fluorescence of 2,7-dichlorofluorescein (DCF). 2'-7'dichlorofluorescein diacetate (DCFH-DA) was added to the medium of cells at a final concentration of $20 \mu \mathrm{M}$ and incubated at $37^{\circ} \mathrm{C}$ for 30 minutes 6 hours following PDT treatment or after the indicated times following PDT treatment. The cells were then washed twice with PBS carefully. Immediately after washing, the cells were measured using a fluorescence spectrophotometer (Varian Australia Pty Ltd) at $488 \mathrm{~nm}$ excitation and $525 \mathrm{~nm}$ emission wavelengths. Experiments were repeated three times independently.

\section{Lipoprotein oxidation determination}

Malondialdehyde (MDA), a stable end product of fatty acid peroxidation, reacts with thiobarbituric acid (TBA) under acidic conditions to form an MDA-TBA adduct with a maximum absorbance at $532 \mathrm{~nm}$. A total of $1.0 \times 10^{5}$ cells were collected and lysed after the treatment. Following the instructions of the MDA detection kit, the lipoprotein oxidation level was determined using a microplate reader (BioTek Elx800, BioTek). 


\section{Measurement of mitochondrial ROS generation}

Three hours following UCNPs-Ce6-PDT treatment, the cells were stained with $20 \mu \mathrm{M}$ DCFH-DA and $1 \mu \mathrm{M}$ MitoSOX (Molecular Probes, Thermo Fisher Scientific, Waltham, MA, USA) for 30 minutes at $37^{\circ} \mathrm{C}$ in a humidified atmosphere in the dark. The cells were then gently washed twice with PBS. Both intracellular and mitochondrial ROS were identified using confocal laser scanning microscopy (CLSM) (LSM 510 Meta; Zeiss, Gottingen, Germany). Both DCF (488 nm excitation wavelength) and MitoSOX (555 nm excitation wavelength) florescence intensities were analyzed using the Zeiss CLSM software (ZEN 2009 Light Edition).

\section{Detection of mitochondrial membrane potential}

MMP was assessed via a jc-1 fluorescent probe (Beyotime Biotechnology, Inc.). Six hours after UCNPs-Ce6-PDT treatment, the macrophages were incubated with $10 \mathrm{mg} / \mathrm{mL}$ jc-1 for 20 minutes at $37^{\circ} \mathrm{C}$ in the dark and were analyzed under the fluorescence microscope. Red-orange fluorescence is attributable to a potential-dependent aggregation in the mitochondria. Green fluorescence, reflecting the monomeric form of jc-1, appeared in the cytosol after mitochondrial membrane depolarization. The alteration of MMP following PDT treatment was also detected using the same method. The fluorescence intensity was measured with a fluorescence spectrophotometer (Varian Australia Pty Ltd) at an excitation wavelength of $488 \mathrm{~nm}$ and emission wavelengths of $530 \mathrm{~nm}$ (green) and $590 \mathrm{~nm}$ (red). Experiments were repeated three times independently.

\section{Detection of MPTP changes}

The intracellular mitochondria were stained specifically with calcein AM through $\mathrm{Co}^{2+}$ quenching of cytosolic calcein fluorescence. About $6 \mathrm{~h}$ after the PDT treatment, the cells were stained with $5 \mu \mathrm{M}$ calcein AM (GenMed Scientifics Inc., Arlington, MA, USA) in the presence of $5 \mathrm{mM}$ cobalt chloride in the dark for 20 minutes at $37^{\circ} \mathrm{C}$, as previously described. ${ }^{21}$ The cells were then carefully washed twice with PBS. Fluorescence was examined using a fluorescence microscope (Olympus IX81, Olympus Corporation), and the change in MPTP following the PDT treatment was also determined using the same method. Calcein AM fluorescence was measured at $488 \mathrm{~nm}$ excitation and $525 \mathrm{~nm}$ emission wavelengths. Experiments were repeated three times independently.

\section{Extraction of mitochondrial and cytosolic fractions}

Both the mitochondrial and cytosolic proteins were isolated using a mitochondria/cytosol fractionation kit (Beyotime Biotechnology, Inc.) according to the manufacturer's instructions. Briefly, cells were harvested, washed twice with PBS, incubated with an ice-cold cytosolic separation buffer containing protease inhibitors, agitated four times for $30 \mathrm{sec}-$ onds, and lysed on ice for 15 minutes. Then, the mixture was subsequently centrifuged at $3,000 \mathrm{rpm}$ for 15 minutes at $4^{\circ} \mathrm{C}$. The supernatant was collected and centrifuged at $12,000 \mathrm{rpm}$ for 30 minutes at $4{ }^{\circ} \mathrm{C}$ to obtain a pellet of mitochondria and a cytosolic supernatant. The pellet was incubated with mitochondrial separation buffer for 30 minutes on ice and then centrifuged at $13,000 \mathrm{rpm}$ for 10 minutes at $4^{\circ} \mathrm{C}$ to obtain the mitochondrial fractions. Both the mitochondrial and cytosolic extracts were used for the analyses of Bax and cytochrome $\mathrm{C}$ by Western blotting.

\section{Western blot analysis}

Sodium dodecyl sulfate-polyacrylamide gel electrophoresis (SDS-PAGE) was performed to analyze the expression of the proapoptotic factor Bax and apoptosis-related proteins, including cytochrome C, procaspase-9, cleaved caspase-9, procaspase-3, cleaved caspase-3, and cleaved poly(ADPribose) polymerase (PARP). After 6 hours of incubation following PDT, the cells were washed twice with PBS and lysed with RIPA buffer on ice for 30 minutes. Then, the cells were harvested and collected via centrifugation at $13,000 \mathrm{rpm}$ for 30 minutes at $4{ }^{\circ} \mathrm{C}$. The bicinchoninic acid protein assay reagent (Beyotime Biotechnology, Inc.) was used to measure the protein concentration in the lysate. Protein samples were mixed with $5 \times$ sample loading buffer (10\% SDS, $5 \% \beta$-mercaptoethanol, $15 \%$ glycerol, $0.01 \%$ bromophenol blue, $200 \mathrm{mM}$ Tris-HCl, $\mathrm{pH}$ 6.7) and separated on a $10 \%-15 \%$ linear SDS-PAGE gel, then transferred to polyvinylidene fluoride membranes (Millipore, MA, USA). Then, the polyvinylidene fluoride membranes were blocked with $5 \%$ nonfat milk in Tris-buffered saline-Tween $20(0.05 \%)$ at room temperature for 2 hours. The membranes were subsequently probed with primary antibodies (against Bax, cytochrome C, procaspase-9, cleaved caspase-9, procaspase-3, cleaved caspase-3, PARP and cleaved PARP; Cell Signaling Technology, Inc., Danvers, MA, USA) overnight at $4^{\circ} \mathrm{C}$. All primary antibodies were diluted 1:1,000. After three washes with Tris-buffered saline-Tween 20, the membranes were incubated with alkaline phosphatase-labeled secondary antibodies at room temperature for 1 hour. The resultant 
immune complexes were detected with enhanced chemiluminescence reagents. All secondary antibodies were diluted 1:1,000. Anti- $\beta$-actin (Cell Signaling Technology, Inc.) was used to confirm equal cytosol loading. Anti-HSP 60 (Cell Signaling Technology, Inc.) was employed to confirm equal mitochondria loading. The percent increases or decreases of protein content were estimated, compared with the vehicle control (100\%).

\section{Statistical analysis}

All experiments were performed independently at least three times. The data were analyzed using one-way ANOVA and are presented as means \pm standard deviation (SD). $P<0.05$ was considered statistically significant.

\section{Results}

\section{Mesoporous-silica-coated nanoparticles as an agent of PDT}

To prepare the UCNPs-Ce6 for the study, we uniformly coated $\mathrm{NaYF}_{4}$ UCNPs with mesoporous silica, in which Ce6 was encased. The irradiation of these UCNPs with a $980 \mathrm{~nm}$ laser resulted in the emission of upconversion visible fluorescence at two primary peaks, namely, green (approximately $540 \mathrm{~nm}$ ) and red (approximately $660 \mathrm{~nm}$ ). We chose this formulation of UCNPs for our PDT experiments because its $660 \mathrm{~nm}$ emission wavelength matched well with the absorption peak of $\mathrm{Ce} 6$ (Figure 1A). To confirm the ability of UCNPs-Ce6 to generate ${ }^{1} \mathrm{O}_{2}$, we used a chemical method using the dye ABDA as an acceptor of ${ }^{1} \mathrm{O}_{2}$. In the presence of ${ }^{1} \mathrm{O}_{2}$, the consumption of ABDA results in fluorescence decay of the dye, thereby providing a means of monitoring ${ }^{1} \mathrm{O}_{2}$ production from UCNPs-Ce6. We measured the extent of ABDA decay in the water in the different treatment groups (Figure 1B). The PDT group demonstrated the highest rate of decay, and this rate was significantly higher than those of control, UCNPs-Ce6 alone, or laser alone, as these groups demonstrated only marginal ABDA decay. To determine whether such decay was a result of the involvement of ${ }^{1} \mathrm{O}_{2}$, we ran a parallel study in which we added the ${ }^{1} \mathrm{O}_{2}$ scavenger $\mathrm{NaN}_{3}$ to the suspension of the PDT group. The presence of $\mathrm{NaN}_{3}$ caused a marked decrease in the rate of ABDA decay, thus confirming the participation of ${ }^{1} \mathrm{O}_{2}$ in this process. Conventional PDT treatment has previously demonstrated low penetration depth, which is an important drawback not only in the treatment of tumors but also in the treatment of AS. We investigated the penetration depth induced by UCNPsCe6-mediated PDT. As shown in Figure 2C, the viability of the THP-1 macrophages treated with PDT $(16 \mu \mathrm{g} / \mathrm{mL}$
UCNPs-Ce6 plus 60 seconds of $980 \mathrm{~nm}$ laser) declined significantly to $67 \%$. However, the addition of pigskin restored cell viability decrease in a thickness-dependent manner, thereby illustrating that the tissues absorbed the $980 \mathrm{~nm}$ laser to a certain extent. The $980 \mathrm{~nm}$ laser penetrated the $6 \mathrm{~mm}$ pigskin and induced cell death. Wang et $\mathrm{al}^{22}$ confirmed that the efficiency of conventional PDT treatment using a $660 \mathrm{~nm}$ laser decreases by $80 \%$ if the laser is blocked by tissues with a thickness of $3 \mathrm{~mm}$, whereas decreases of only $5 \%$ is noted in the setting of NIR-treated PDT. We also indirectly detected the accumulation of UCNPs-Ce6 in the THP-1 macrophages via the fluorescence of $\mathrm{Ce} 6$ (red color), and we observed that the fluorescence reached its highest level at 4 hours following incubation (Figure 1D).

\section{Cell viability with different treatments}

The CCK-8 assay demonstrated that cell viability decreased with increasing UCNPs-Ce6 concentrations (Figure 2A) and laser irradiation times (Figure 2C), and the cell viability was significantly decreased at a concentration of $32 \mu \mathrm{g} / \mathrm{mL}$ and a laser irradiation time of 90 seconds. However, cell viability was not significantly affected in the cultures treated with $16 \mu \mathrm{g} / \mathrm{mL}$ UCNPs-Ce6 with increasing incubation times (Figure 2B). High concentrations of UCNPs-Ce6 and long laser irradiation time periods at strong intensities may result in cell necrosis, which subsequently results in the augmentation of the necrotic core and plaque vulnerability, ${ }^{23}$ thereby increasing the risk of acute cardiovascular disease. Clinically, apoptosis is preferred over necrosis because it triggers fewer inflammatory reactions. ${ }^{24}$ Therefore, it was important to identify the therapeutic conditions that resulted in the lowest necrotic ratio. Among the cultures treated with different UCNPs-Ce6 concentrations $(2-16 \mu \mathrm{g} / \mathrm{mL})$ for 60 seconds of laser irradiation, cell viability decreased with increasing drug concentrations (Figure 2D). When the cells were incubated with $16 \mu \mathrm{g} / \mathrm{mL}$ UCNPs-Ce6 and subjected to different durations of laser irradiation, cell viability decreased with increasing time (Figure 2E). These two results indicated that the cytotoxic effects were enhanced by the combination of increased UCNPs-Ce6 concentrations and laser irradiation. The cell survival rates also decreased significantly following PDT (16 $\mu \mathrm{g} / \mathrm{mL}$ UCNPs-Ce6 and 60 seconds of laser irradiation) with different capacities $(0.5-2.0 \mathrm{~W})$ (Figure 2F). Therefore, the application of $16 \mu \mathrm{g} / \mathrm{mL}$ UCNPs-Ce6 and 60 seconds of laser irradiation with a capacity of $2.0 \mathrm{~W}$ represented the optimal conditions under which PDT maximized the decrease in cell viability (Figure 2G). 


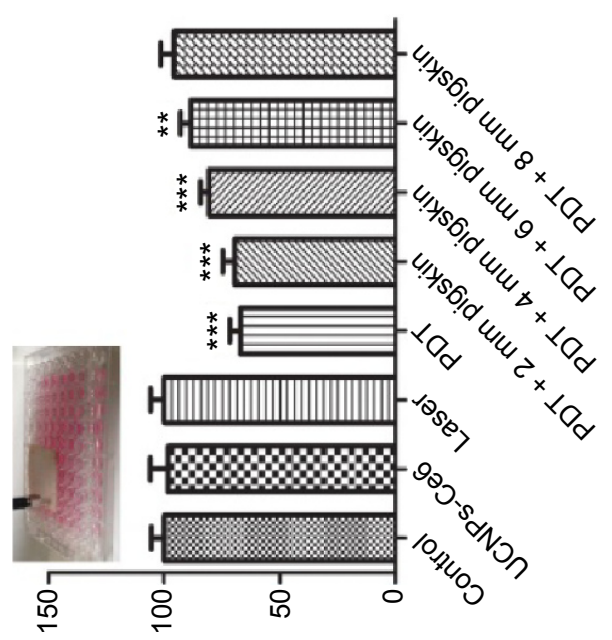

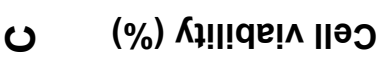

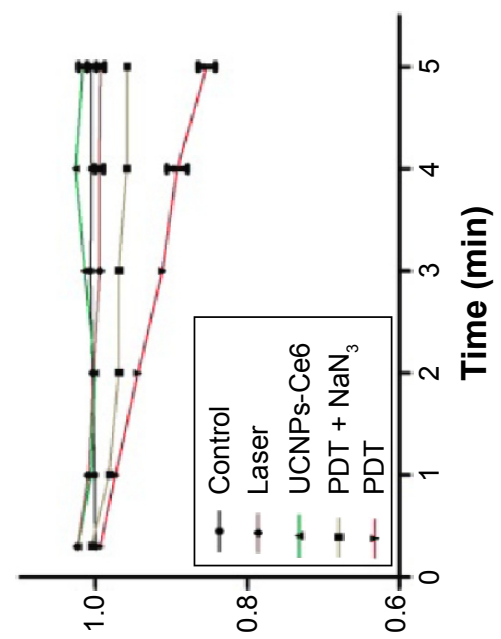

(s $8 \mathrm{~L}=\mathfrak{l}$ 의 pəz!|lemsou)

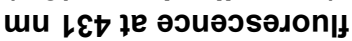

$m \quad$ yeəd әКр $\forall a q \forall$
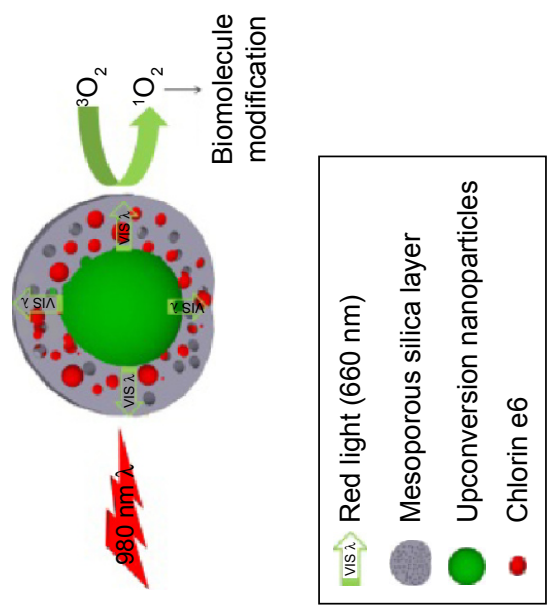

$\varangle$

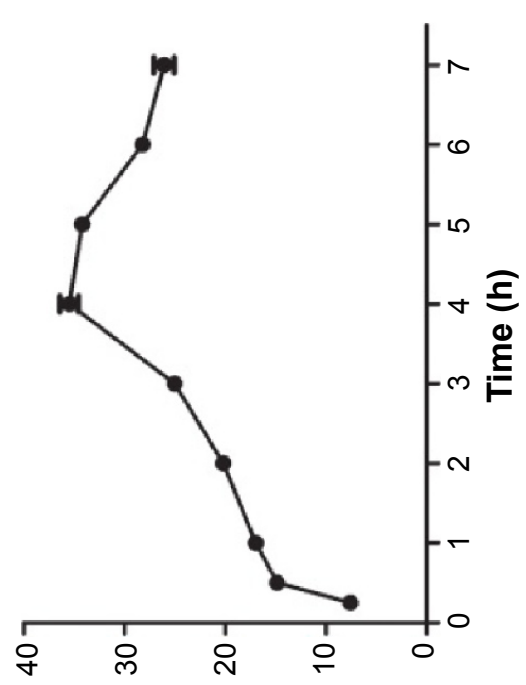

(ne) Kł!

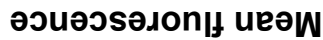

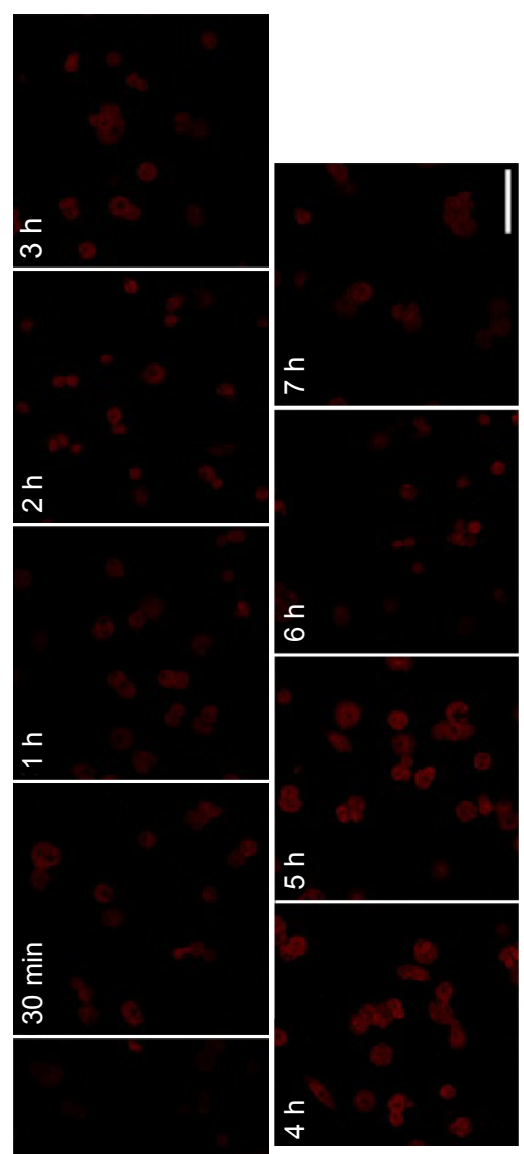

톨

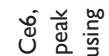

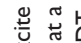

×

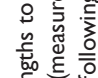

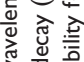

嵌

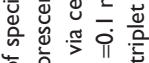

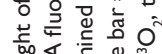

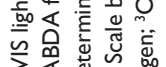

웡

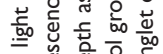

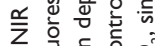

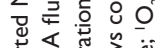

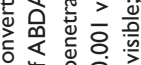

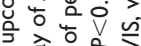

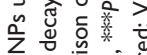

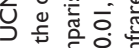

온

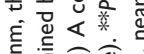

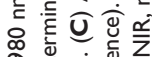

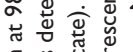

등

可产.

on. 운

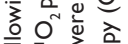

난.

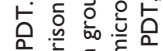

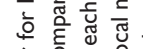

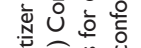

鱼品

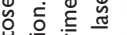

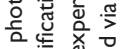

造 :

응 $\frac{0}{3} \div$

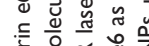

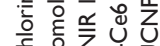

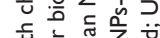

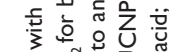

웡

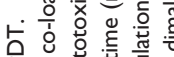

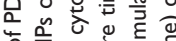

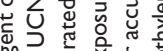

年

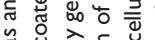

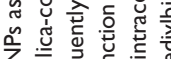

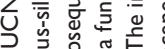

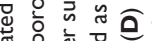

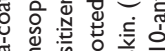

كै

它语

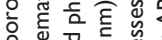

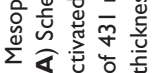

ming 
A

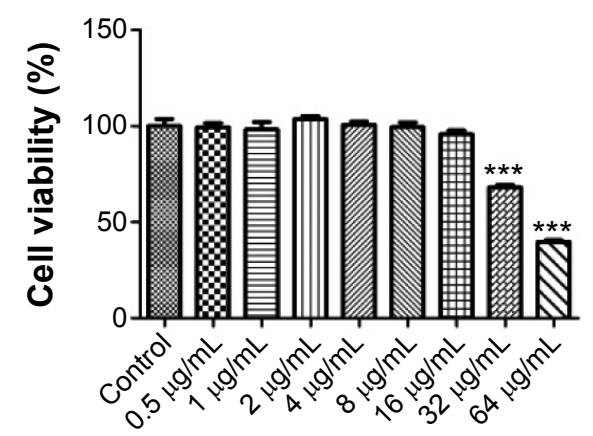

C

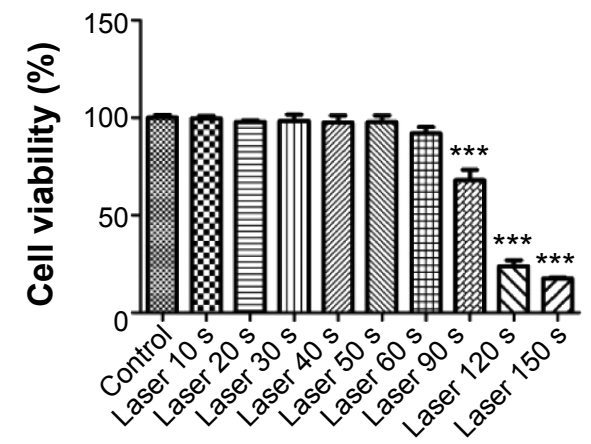

E

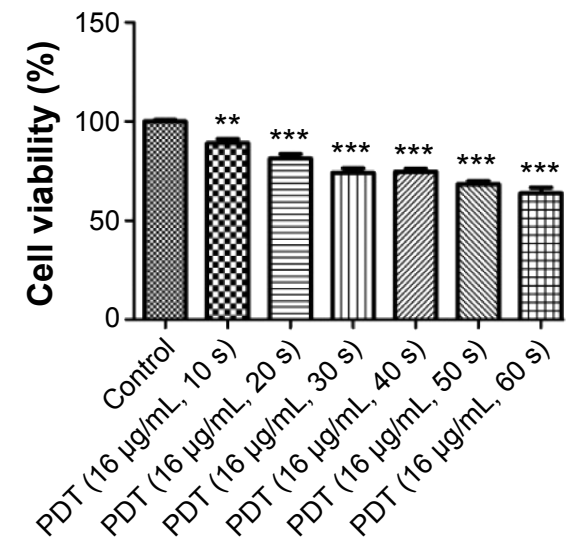

B
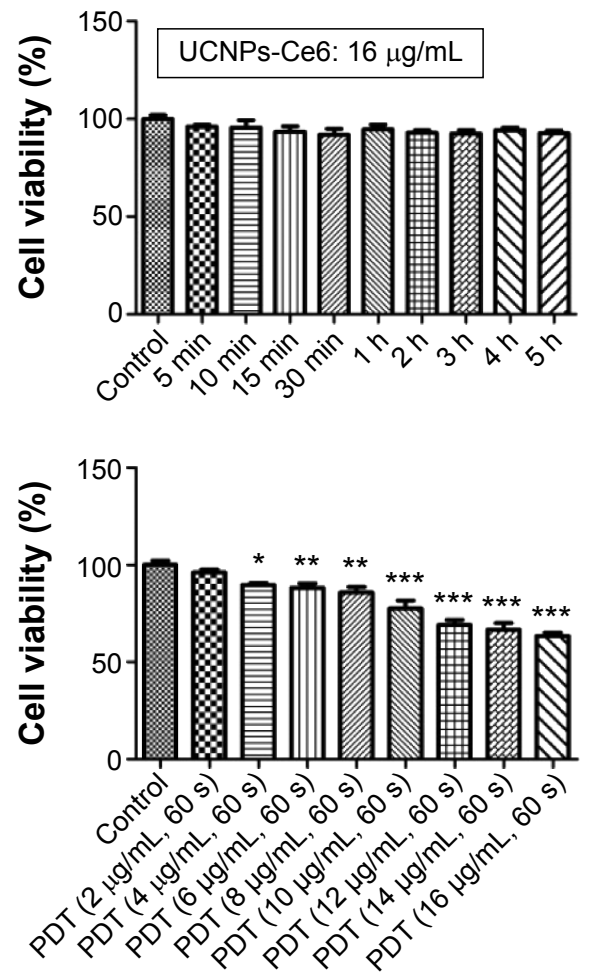

$\mathbf{F}$

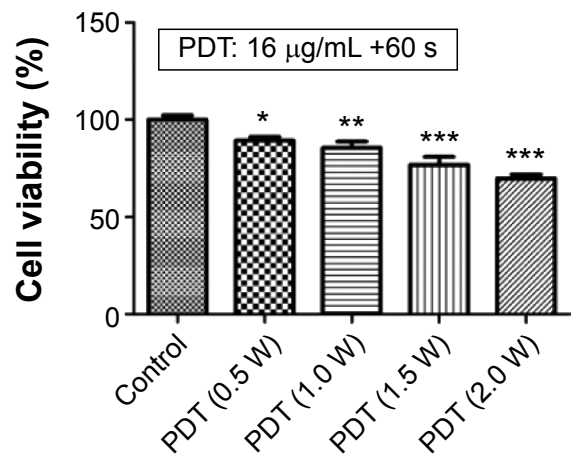

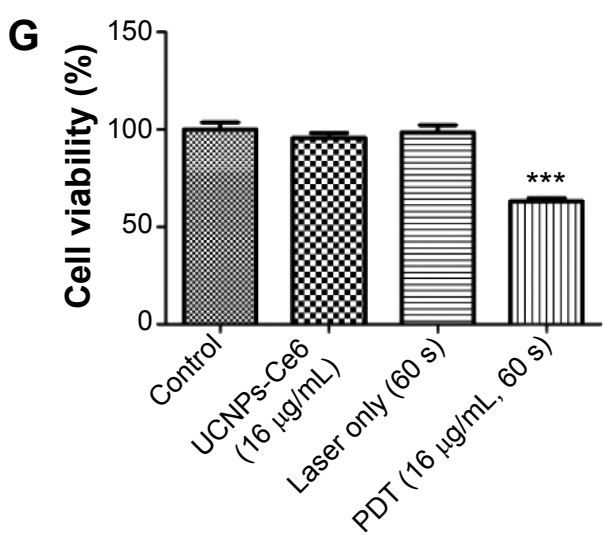

Figure 2 The survival rates of THP-I macrophages as determined via a CCK-8 assay.

Notes: (A) The survival rates of THP-I macrophages following exposure to different UCNPs-Ce6 concentrations for $4 \mathrm{~h}$. (B) The survival rates of THP-I macrophages following incubation with $16 \mu \mathrm{g} / \mathrm{mL}$ UCNPs-Ce6 for different time intervals. (C) The survival rates of THP-I macrophages following different laser irradiation time intervals. (D) The survival rates of THP-I macrophages following $60 \mathrm{~s}$ of laser irradiation with different UCNPs-Ce6 concentrations. (E) The survival rates of THP-I macrophages following different laser irradiation time intervals with $16 \mu \mathrm{g} / \mathrm{mL}$ UCNPs-Ce6. (F) The survival rates of THP-I macrophages following exposure to different laser densities at $16 \mu \mathrm{g} / \mathrm{mL}$ UCNPs-Ce6. (G) The survival rates of THP-I macrophages following different treatments. $* P<0.05$, $* * P<0.01$, $* * * P<0.001$ vs control group.

Abbreviations: UCNPs, upconversion nanoparticles; Ce6, chlorin e6; PDT, photodynamic therapy; h, hours. 
Apoptosis of THP-I macrophages induced by UCNPs-Ce6-mediated PDT

Previous investigations ${ }^{25-27}$ have demonstrated that PDT induces both apoptosis and necrosis depending on the drug concentrations as well as the laser irradiation time and intensity. In this study, we evaluated the type of cell death that occurred following UCNPs-Ce6-mediated PDT using three different methods. The Hoechst 33258 and PI assays for cell apoptosis and necrosis are shown in Figure 3A. Nearly equal cell necrosis rates were noted in the UCNPs-Ce6 and PDT groups, whereas the rates of necrosis in the control and laser groups were low, which indicated that UCNPs-Ce6 exerts toxic effects on THP-1 macrophages and that high drug concentrations and laser irradiation may kill cells directly via necrosis. Clinically, apoptosis is preferred over necrosis in the macrophages associated with atherosclerotic plaques as necrosis may precipitate an inflammatory reaction and disrupt plaque homeostasis. ${ }^{24}$ Therefore, it is essential that optimal conditions for PDT treatment are used. The apoptosis rates between the UCNPs-Ce6 and laser groups were similar. However, the PDT group exhibited a significant increase in the rate of apoptosis.

We evaluated the effectiveness of the treatment in terms of apoptotic cell death by performing TUNEL staining on the THP-1 macrophages from different treatment groups at 6 hours after the PDT treatment. We identified a larger population of apoptotic cells in the PDT group, which were characterized by TUNEL-positive staining (green) and the nuclear fragmentation (blue) in Figure 3B. However, the degrees of apoptosis in both the UCNPs-Ce6 and laser groups were not significantly different from that in the control group. We also demonstrated that UCNPs-Ce6-mediated PDT promoted caspase-dependent apoptosis in the THP-1 macrophages. As shown in Figure 3C, PDT resulted in the increased expression of cytochrome $\mathrm{C}$, which was accompanied by procaspase- 9 cleavage into active caspase-9. In addition, the activation of caspase-3 and the cleaved product of PARP were detected in the PDT group. The results of the cell viability tests indicated that the broad-spectrum caspase inhibitor, z-VAD-FMK, prevented the cell death of the THP-1 macrophages in a dose-dependent manner (Figure 3D). These findings suggested that activation of caspase-related proteins after PDT treatment could be the key to induce apoptosis in THP-1 macrophages.

\section{ROS generation induced by UCNPs-Ce6- mediated PDT}

Photosensitization, a process induced by highly noxious reactive species, such as free radicals and ROS generated by photosensitizers following activation, is the primary mechanism by which cellular damage is induced following PDT treatment. ${ }^{28}$ Evidence has suggested that oxidative stress is a potential common mediator of apoptosis. Therefore, the generation of ROS, particularly ${ }^{1} \mathrm{O}_{2}$ molecules following PDT treatment, plays a key role in regulating the efficiency of photosensitization. ${ }^{29}$

Results (Figure 1B) have confirmed that UCNPs-Ce6 is capable of generating ${ }^{1} \mathrm{O}_{2}$ after receiving $980 \mathrm{~nm}$ laser irradiation. Therefore, we examined the production of ROS in live cells. As compared with the control group, the green fluorescence increased significantly in the PDT group at 6 hours following treatment, whereas slight increases in the green fluorescence were observed in both the UCNPs-Ce6 group and the laser group (Figure 4A). This fluorescence was inhibited by the addition of the ROS scavenger, NAC, and the singlet scavenger, $\mathrm{NaN}_{3}$. We investigated the cause of the ROS alteration within 6 hours following PDT treatment, which was important in the study of apoptosis following PDT. We also evaluated the time course of ROS generation following PDT. Interestingly, we observed that ROS generation increased within the first 3 hours before decreasing with time (Figure 4B). The time course of ROS generation indicated that PDT increased the level of ROS, which peaked at 3 hours. The initial increase in ROS generation was most likely due to the reactivity of the ${ }^{1} \mathrm{O}_{2}$ formed early following PDT and its ability to induce the formation of other ROS species that were detected via DCFH-DA (DCFH-DA does not detect ${ }^{1} \mathrm{O}_{2}$ but reacts with hydroperoxides). To determine which chemical was more efficient in altering PDT-induced apoptosis, several other free radical scavengers, including mannitol, $\mathrm{SOD}, \mathrm{CAT}$, and $\mathrm{NaN}_{3}$, were tested prior to treatment. As shown in Figure 4C, both mannitol and $\mathrm{NaN}_{3}$ exerted strong effects in preventing the decline in cell viability. These results indicated that the hydroxyl radical may be the primary ROS that decreases cell viability, whereas ${ }^{1} \mathrm{O}_{2}$ may act as a trigger for the induction of an imbalance in ROS generation in THP-1 macrophages (Figure 4C). A previous study demonstrated that the accumulation of ROS in tumor cells may cause oxidizing reactions in biomolecules that disrupt the integrity of both lipid membranes and proteins. ${ }^{30}$ We measured MDA levels and observed that in both the UCNPs-Ce6 and the laser groups, the MDA levels were slightly increased compared with the control group (Figure 4D). The MDA level in the PDT group was significantly increased as it was almost three times that in the control group. However, the MDA level in the PDT group decreased following pretreatment with NAC. 


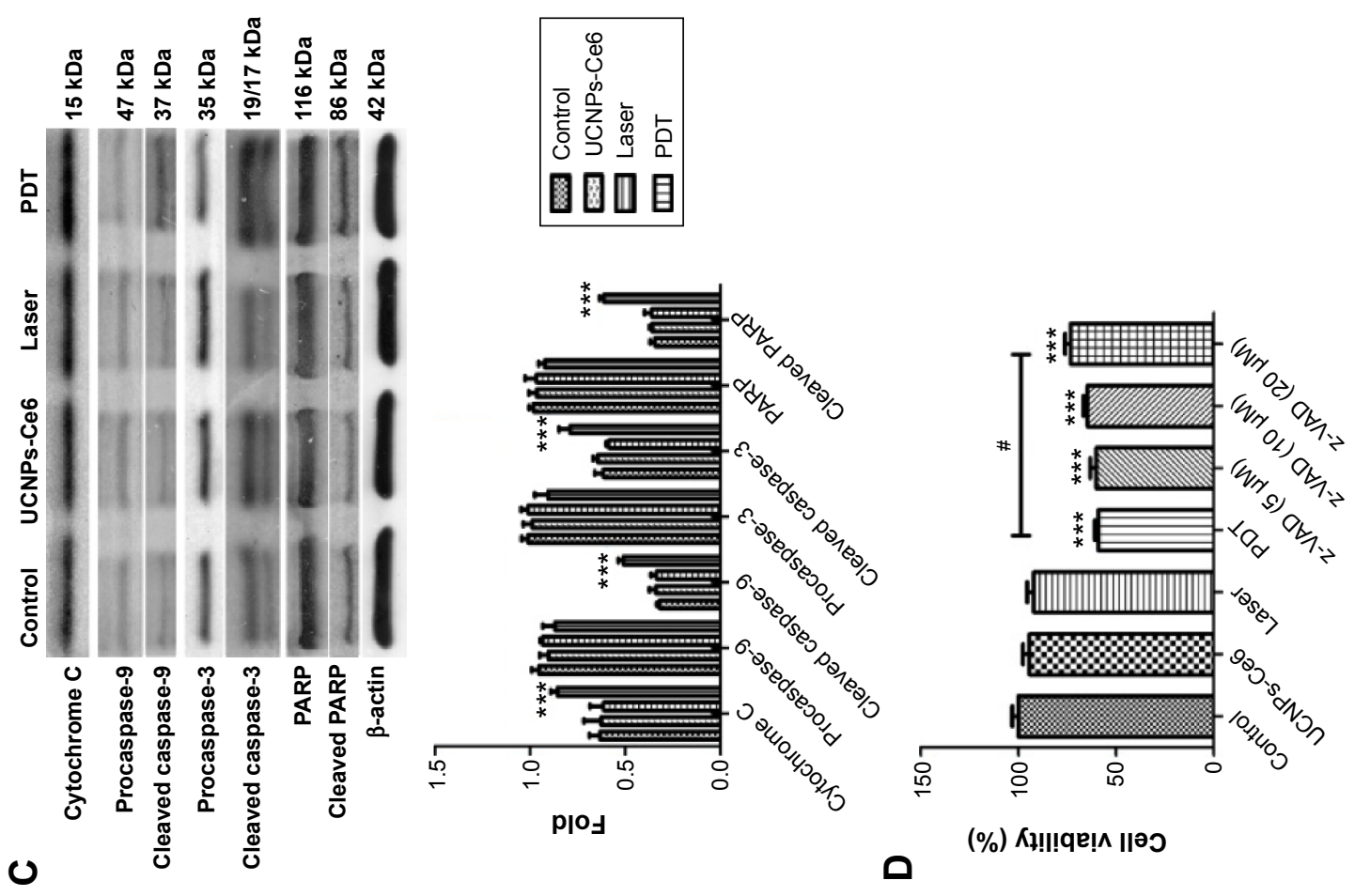

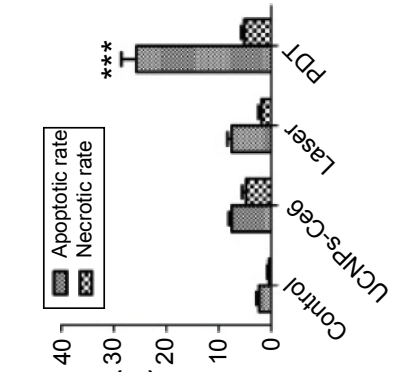

(\%) әреג

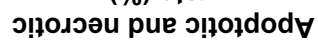

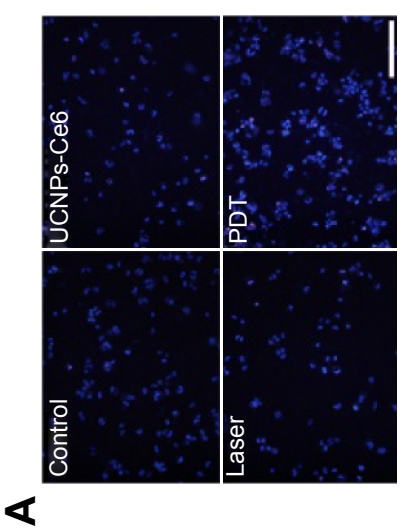

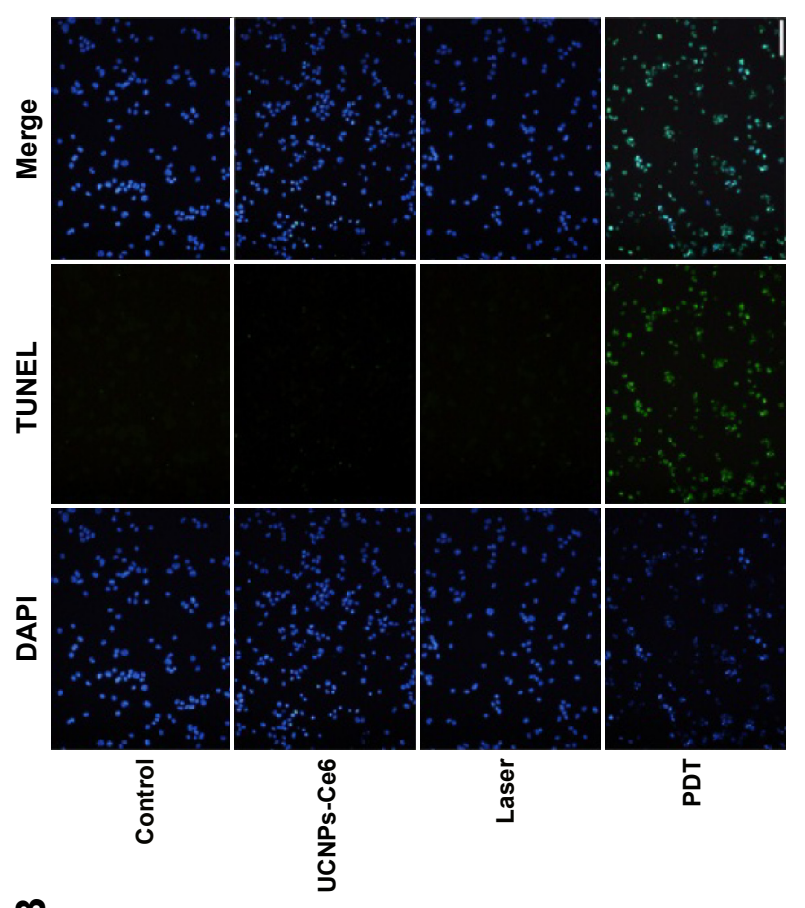

뭉 $\frac{1}{0} \frac{n}{\overline{0}}$

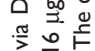

它密

उ $\overline{0}$

है

过

至

乙U.

入े

过

品气

뭉ㅇㅎㅇ

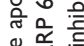

定

है 过 II

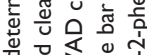

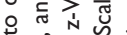

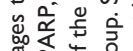

के के के

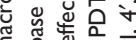

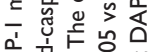

圭 总

no

它

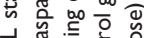

㟧立。行

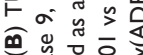

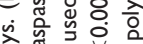

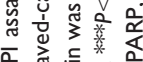

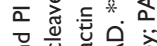

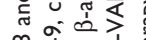

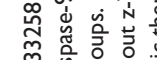

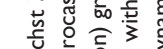

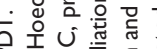

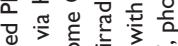

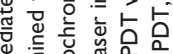

हो

U⿺辶寸

离

ป

入

ญัّ

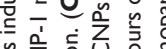

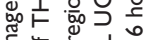

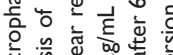

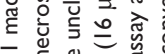

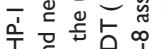

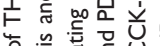

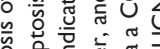

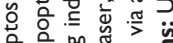

这紫

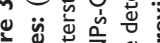

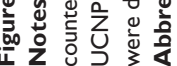


A

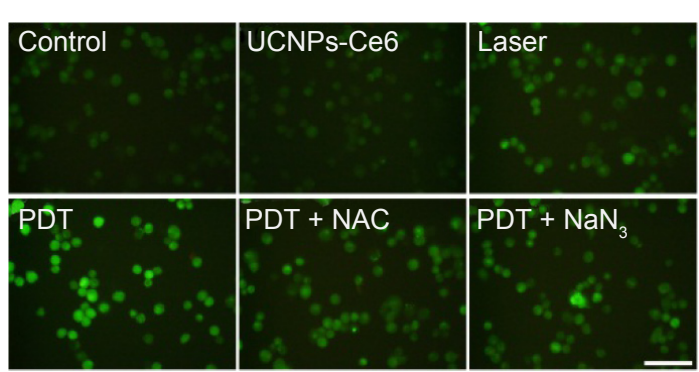

B

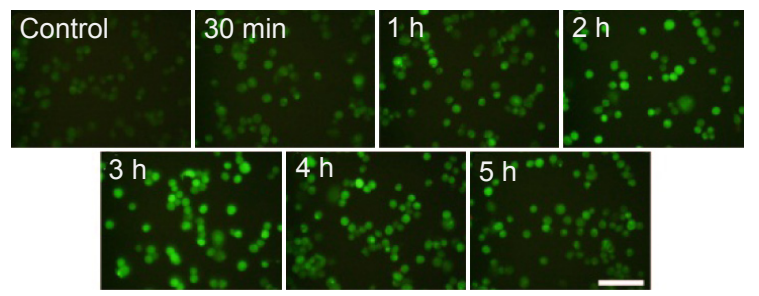

C

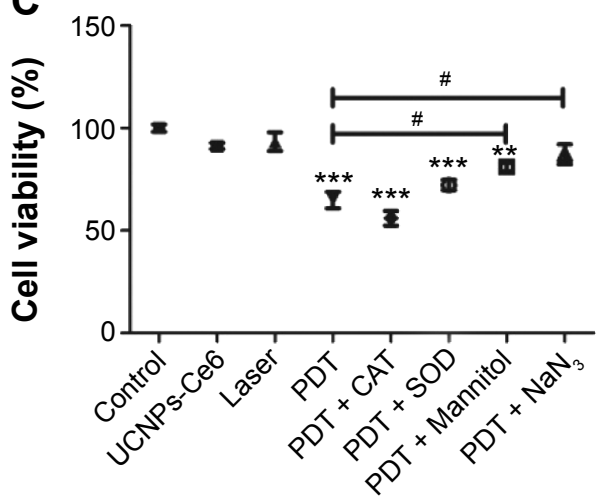

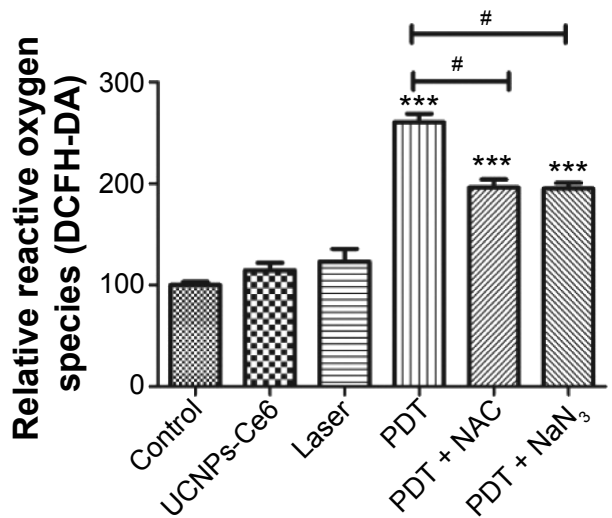

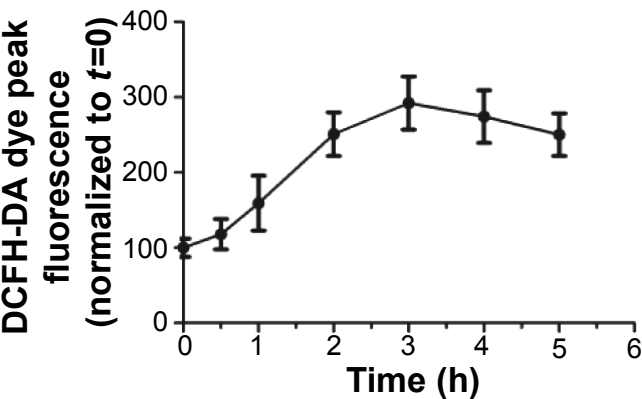

D

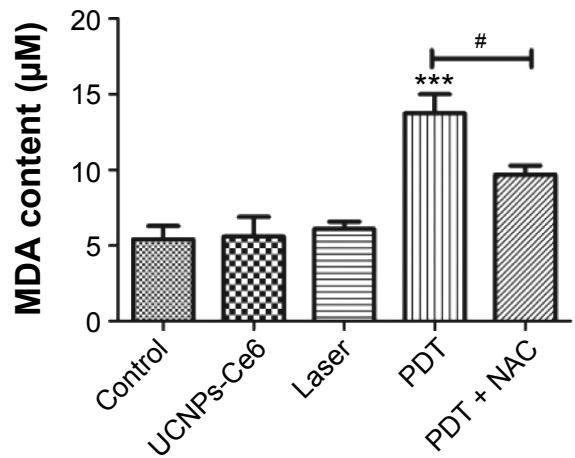

Figure 4 Relative reactive oxygen species (ROS) generation induced by UCNPs-Ce6-mediated PDT.

Notes: (A) Fluorescent photomicrographs of THP-I macrophages stained using DCFH-DA, indicating intracellular ROS generation. (B) The alteration of ROS following PDT. (C) The effect of active oxygen scavengers on cell viability following PDT. To determine the primary inducer of apoptosis following PDT, several free radical scavengers, including $200 \mathrm{U} / \mathrm{mL}$ catalase (CAT, a scavenger of hydrogen peroxide), $100 \mathrm{mg} / \mathrm{mL}$ superoxide dismutase (SOD, a scavenger of superoxide anion radicals), $100 \mathrm{mM}$ mannitol (a scavenger of the hydroxyl radical), and $10 \mathrm{mM}$ sodium azide $\left(\mathrm{NaN}_{3}\right.$, a scavenger of singlet oxygen molecules), were employed prior to treatment. Cell viability was examined via a CCK-8 assay. (D) MDA content following UCNPs-Ce6-mediated PDT. $* * P<0.00$ I vs control group. ${ }^{* * *} P<0.00$ I vs control group. ${ }^{\# P<0.05}$ vs PDT group. Scale bar $=0.1 \mathrm{~mm}$.

Abbreviations: UCNPs, upconversion nanoparticles; Ce6, chlorin e6; PDT, photodynamic therapy; DCFH-DA, 2'-7'-dichlorofluorescein diacetate; NAC, N-acetyl-Lcysteine; MDA, malondialdehyde; $h$, hours.

\section{Collapse of mitochondrial membrane potential $(\psi \mathrm{m})$ induced by UNCPs- Ce6-mediated PDT}

The aforementioned results indicate the necessity of identifying the cellular targets associated with PDT. Emerging evidence that the mitochondria participate in a number of functions, including ATP production, amino acid biosynthesis, specific ion buffering, the management of ROS, and the initiation of apoptosis, has demonstrated that mitochondria play a critical role in cell death signaling pathways. ${ }^{31-34}$ We subsequently measured both mitochondrial ROS production and MMP $(\psi \mathrm{m})$ in the THP-1 macrophages following PDT treatment. ROS production in the mitochondria increased significantly in the PDT group as determined via MitoSOX. After intracellular ROS generation was blocked by NAC, mitochondrial ROS production returned to its normal state. This phenomenon was confirmed by the appearance of $\mathrm{NaN}_{3}$.

As shown in Figure 5A, the normal THP-1 macrophages exhibited red-orange fluorescence, and neither irradiation alone nor UCNPs-Ce6 alone triggered an MMP change. 

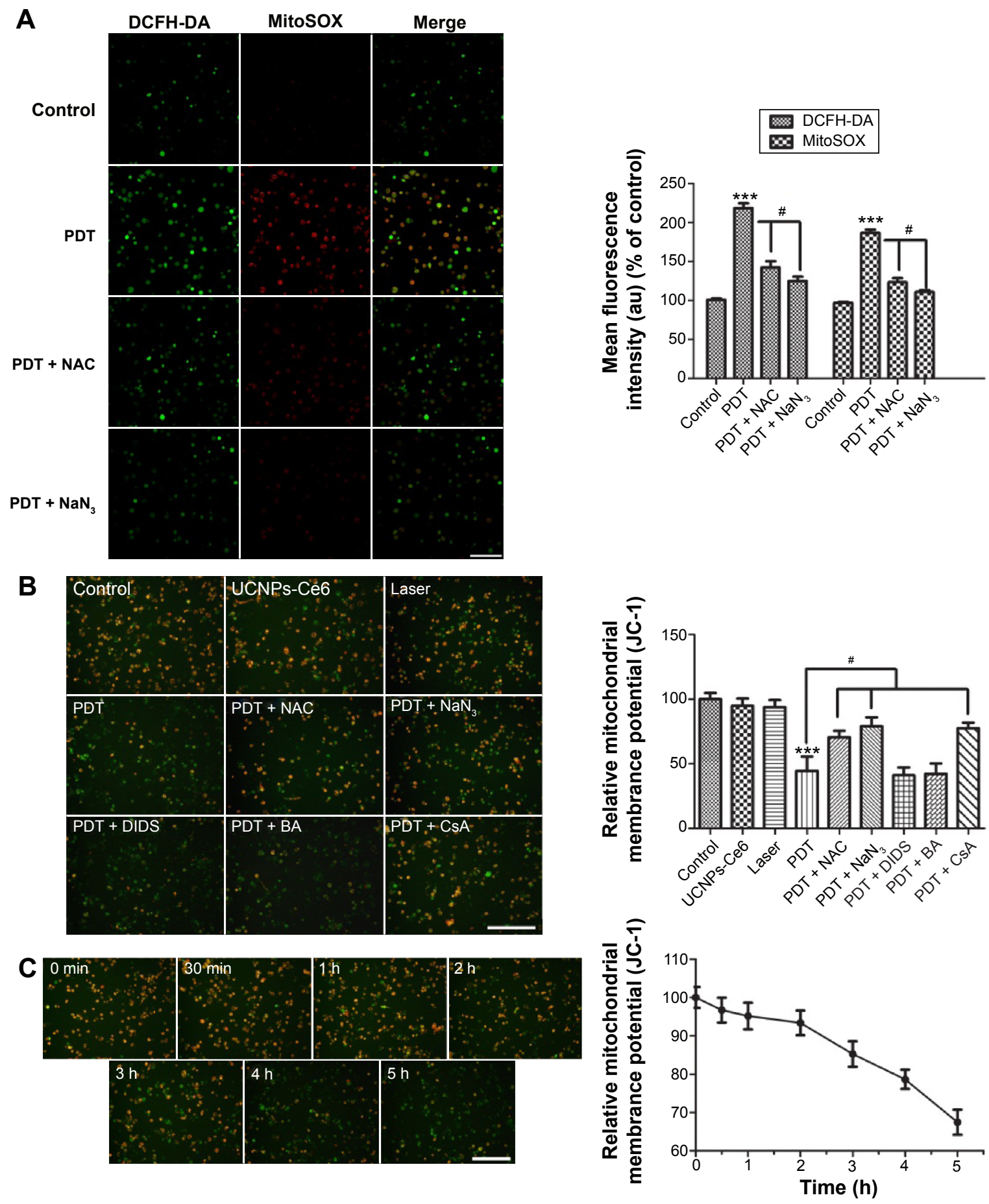

Figure 5 Mitochondrial ROS bursts and mitochondrial membrane potential (MMP) loss in THP-I macrophages induced by UCNPs-Ce6-mediated PDT.

Notes: Fluorescent photomicrographs of THP-I macrophages (A) stained with MitoSOX, depicting the mitochondrial ROS increases; (B) stained with JC-I to depict the MMP; (C) depicting the decrease in MMP with time following PDT. $* * * P<0.001$ vs control group; ${ }^{P}<0.05$ vs PDT group. Scale bar $=0.1$ mm.

Abbreviations: UCNPs, upconversion nanoparticles; Ce6, chlorin e6; PDT, photodynamic therapy; DCFH-DA, 2'-7'-dichlorofluorescein diacetate; NAC, N-acetyl-Lcysteine; DIDS, disulfonic acid disodium; BA, bongkrekic acid; CsA, cyclosporin A; ROS, reactive oxygen species; $h$, hours.

The THP-1 macrophages developed a diffuse green staining pattern following PDT treatment, which was indicative of decreased MMP. However, after adding the scavengers (NAC and $\mathrm{NaN}_{3}$ ), the decrease in MMP was prevented. Emerging studies have demonstrated that the opening of the
MPTP is involved in the mitochondrial-dependent apoptotic pathway. ${ }^{35}$ Inhibitors (BA, DIDS, and CsA) of different subunits of the MPTP, including adenine nucleotide translocase, voltage-dependent anion channel (VDAC), and cyclophilin-D (CYPD), ${ }^{36,37}$ were utilized to analyze the functions of those 
subunits in regulating the MMP following PDT treatment. CsA was found to be involved in preventing the loss of MMP, indicating that CYPD may play a vital role in regulating changes in MMP (Figure 5B). Alterations in MMP following PDT treatment were also analyzed. The MMP began to decrease when the THP-1 macrophages received the PDT treatment, and this decrease was gradual within the first 2 hours before becoming more precipitous (Figure 5C).

\section{Release of cytochrome C via MPTP opening induced by UCNPs-Ce6- mediated PDT}

To determine whether the MPTP participated in the apoptotic mechanism induced by UCNPs-Ce6-mediated PDT, changes in MPTP activity were assessed by calcein AM staining. The green fluorescence intensity decreased in a time-dependent manner (Figure 6C). The relative MPTP fluorescence level in the PDT group decreased as the incubation time following PDT treatment increased. To confirm which of the MPTP subunits was involved in this process, we used different subunit inhibitors. The results indicated that the green fluorescence intensity was lowest in the PDT group and was increased by the addition of the MPTP inhibitors, DIDS, BA, and CsA (Figure 6A, B). After adding the CYPD inhibitor, CsA, the fluorescence level increased significantly compared with the PDT group. However, the BA inhibitor also prevented the decrease in fluorescence, but this change was not significant compared with the PDT group. Therefore, we concluded that UCNPs-Ce6-mediated PDT induced the MPTP opening via the regulation of the primary functional site, CYPD. In response to most kinds of stress, the tripartite Bcl-2 family of proteins governs commitment of cells to apoptosis. ${ }^{38} \mathrm{We}$ analyzed the expression of the proapoptotic factor, Bax, and cytochrome $\mathrm{C}$ in the mitochondria and cytosol. PDT caused a significant increase in mitochondria-localized Bax and a corresponding decrease in cytosolic Bax, whereas the opposite pattern was observed for cytochrome $\mathrm{C}$, which decreased in the mitochondria and increased in the cytosol (Figure 6D). Interestingly, CsA inhibited the release of cytochrome $\mathrm{C}$ as opposed to inhibiting Bax translocation.

\section{Blockage of Bax translocation and release of cytochrome $C$ by NAC post-PDT}

Bax translocation from the cytosol to the mitochondria acts as a trigger of outer mitochondrial membrane permeabilization, and apoptotic proteins release in PDT was reported in previous studies. ${ }^{39}$ As we observed that UCNPs-Ce6-mediated PDT induced both Bax translocation and cytochrome $\mathrm{C}$ release, we investigated whether this phenomenon was induced by ROS following PDT treatment. We subsequently assessed the contribution of ROS to this particular process following PDT via fluorescence microscopy. Figure 7A shows that at 6 hours following PDT treatment, diffuse yellow fluorescence resulted from the overlap of green fluorescence and red fluorescence, thus indicating the translocation of Bax from the cytosol to the mitochondria, and Bax was closely anchored to the cytosol in both the control group and the PDT group pretreated with NAC. Additionally, the accumulation of cytochrome $\mathrm{C}$ within the mitochondria before diffusing into the cytosol at 6 hours following PDT treatment was also observed, whereas the cytochrome $\mathrm{C}$ in the PDT group pretreated with NAC reverted to its initial state. Moreover, the blockade of Bax translocation and cytochrome $\mathrm{C}$ release by NAC was analyzed via Western blotting (Figure 7B, C). UCNPs-Ce6-mediated PDT triggered the translocation of Bax to the mitochondria from the cytosol in a time-dependent manner. This process was accompanied by cytochrome $\mathrm{C}$ release from the mitochondria to the cytosol. This process was prevented by NAC, which indicated that ROS may be an exogenous initiator of this biological process in the mitochondria following induction by UCNPs-Ce6-mediated PDT.

\section{Discussion}

AS is a leading cause of death in industrialized societies and worldwide, ${ }^{40}$ and it is characterized by the formation of vulnerable plaques in which macrophage foam cells accumulate to expand the subendothelial layer and its thin fibrous cap. Once the vulnerable plaques rupture under stress, it will trigger acute thrombotic vascular disease, including myocardial infarction, stroke, and sudden cardiac death. ${ }^{3}$ Therefore, a key step in the reduction of the formation of these plaques is the clearance of macrophage foam cells. PDT utilizes a combination of chemical photosensitizers, light, and molecular oxygen and has long been used in the treatment of cancer. Our group has previously confirmed that 5 -aminolevulinic-acidmediated PDT retarded the progression of AS by reducing both the numbers and the content of plaques, ${ }^{10}$ which was consistent with the results of the study by Waksman et $\mathrm{al}^{9}$ who observed that MV0611-mediated PDT decreases plaque inflammation and attenuates the progression of the disease. However, conventional PDT using VIS light has an important weakness, as the absorbance of VIS light by biomolecules results in low penetration efficiency because this form of light cannot pass through thick tissue. As most biomolecules absorb minimal amounts of light in the NIR window, the 


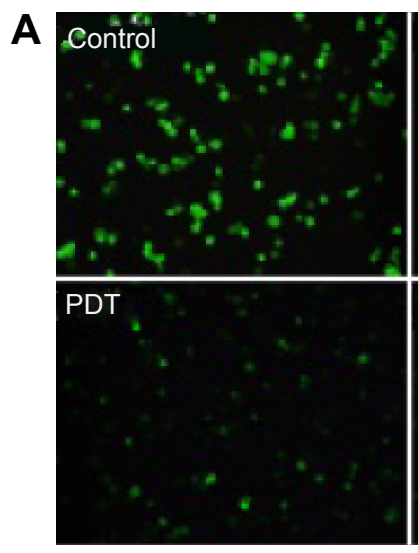

B

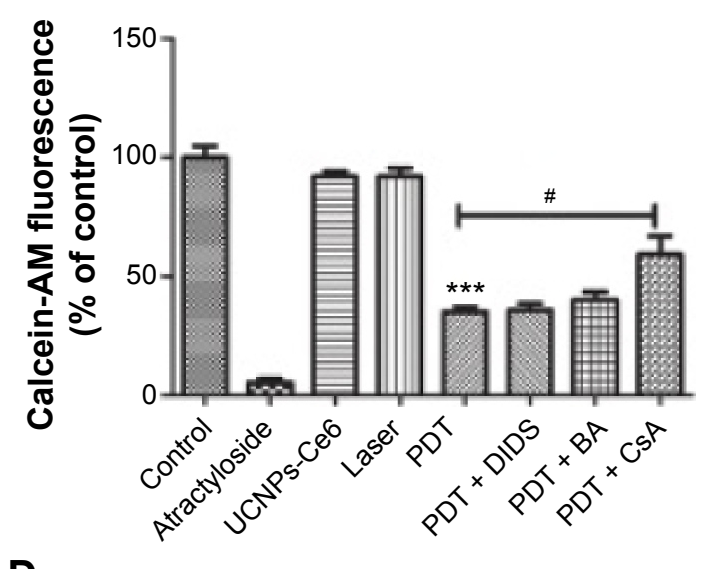

D
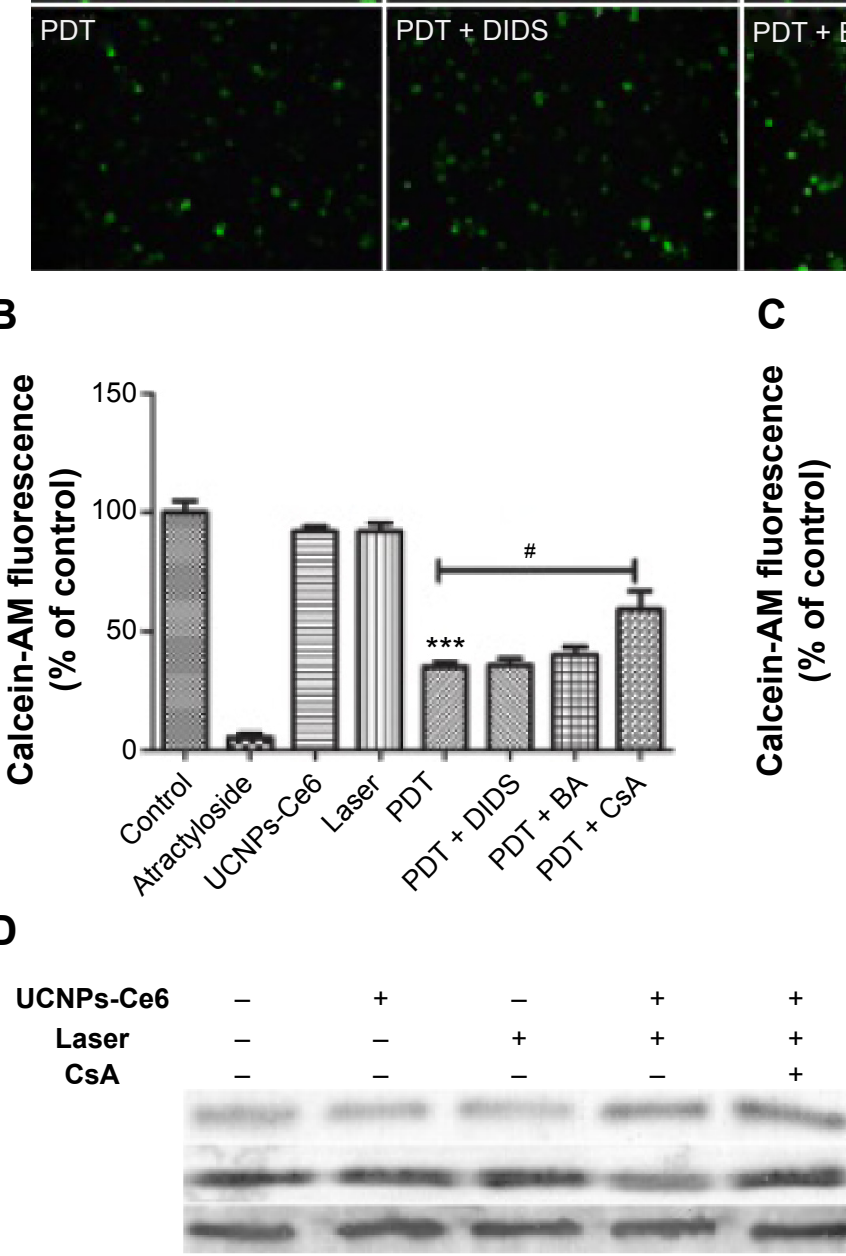

C
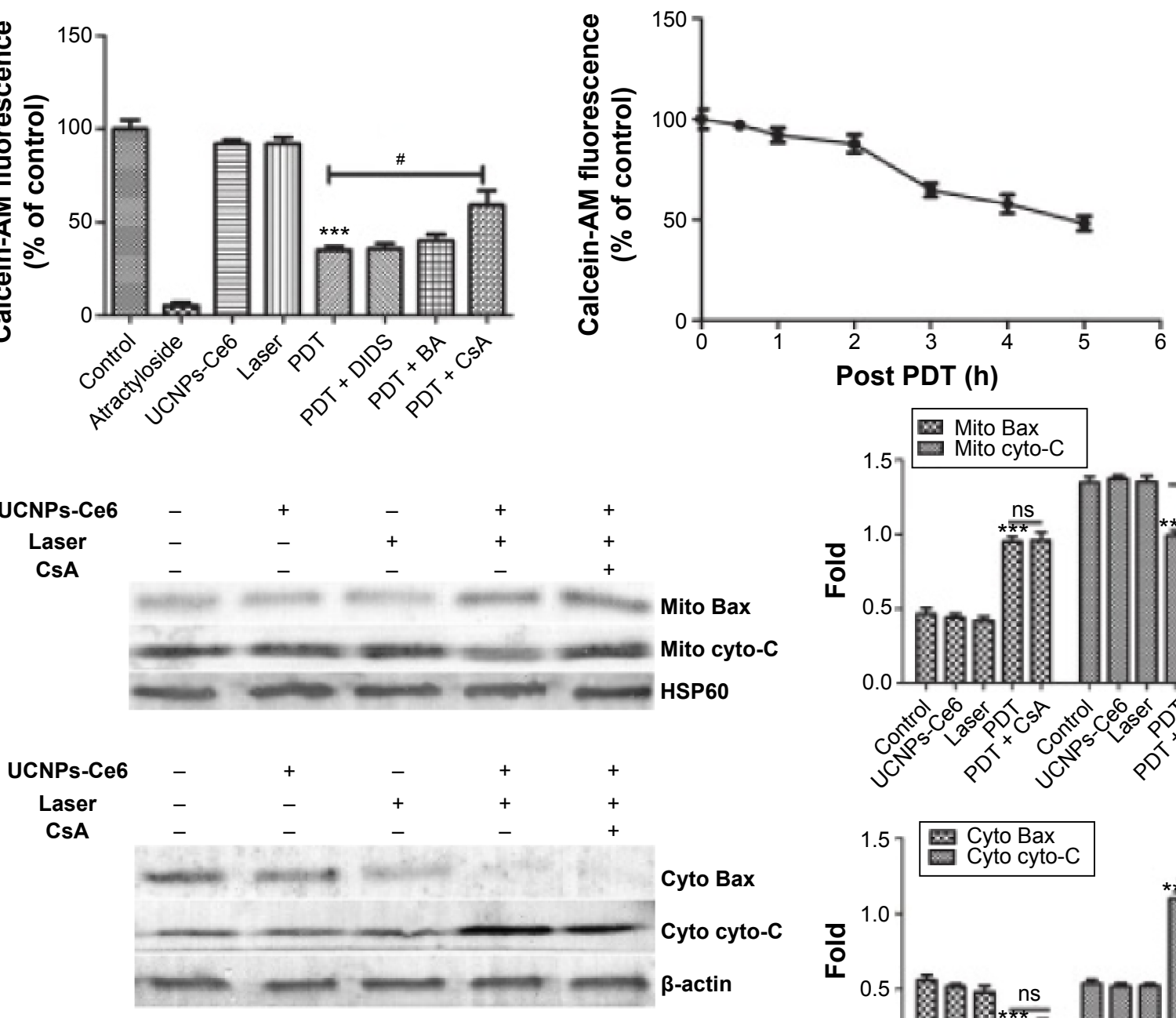
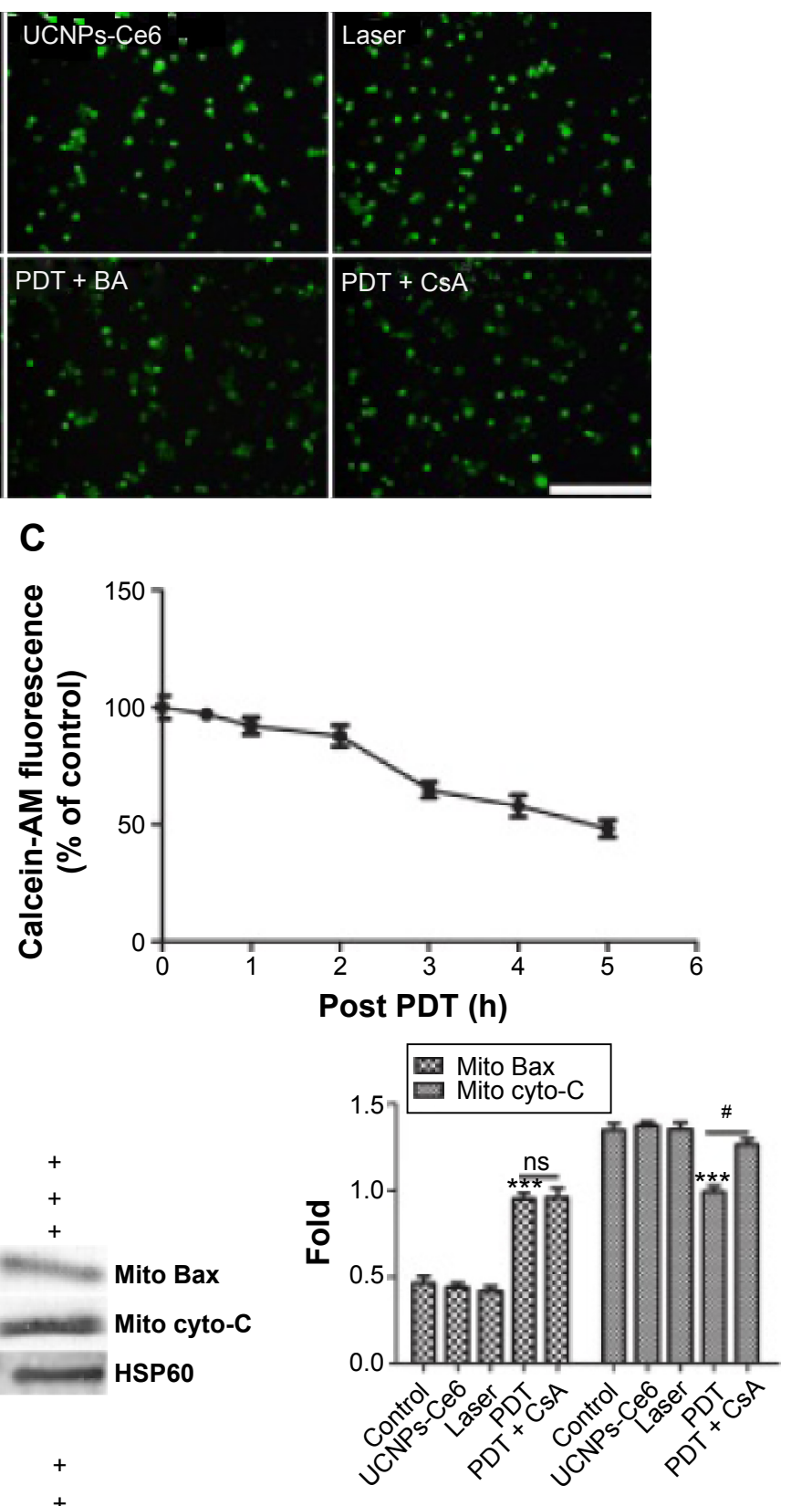

Cyto Bax

Cyto cyto-C

$\beta$-actin

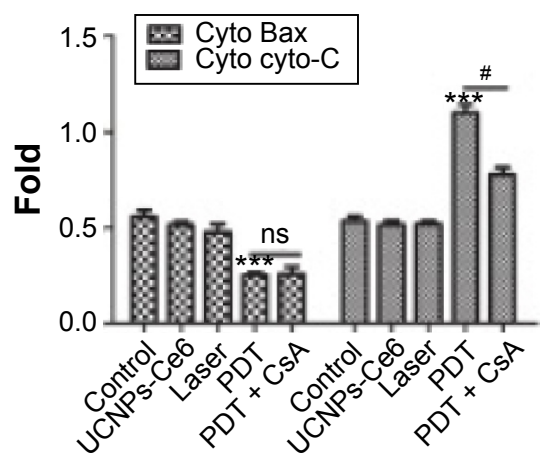

Figure 6 Bax translocation and the release of cytochrome C via opening of the MPTP, which was induced by UCNPs-Ce6-mediated PDT.

Notes: (A) Fluorescent photomicrograph of mitochondrial permeability transition pore activation via calcein $\mathrm{AM} / \mathrm{CoCl}{ }^{2+}$ labeling. (B) The mean fluorescence intensity of the calcein AM levels of THP-I macrophages as determined using a fluorospectrophotometer. (C) The decrease in calcein AM intensity following PDT. (D) Bax translocation and the release of cytochrome $C 6$ hours after PDT via Western blotting. ${ }^{*} * * P<0.001$ vs control group; ${ }^{*} P<0.05$ vs $P D T$ group. Scale bar $=0.1$ mm.

Abbreviations: UCNPs, upconversion nanoparticles; Ce6, chlorin e6; PDT, photodynamic therapy; DIDS, disulfonic acid disodium; BA, bongkrekic acid; CsA, cyclosporin A; MPTP, mitochondrial permeability transition pore; cyto-C, cytochrome C; h, hours; ns, no significance. 
A

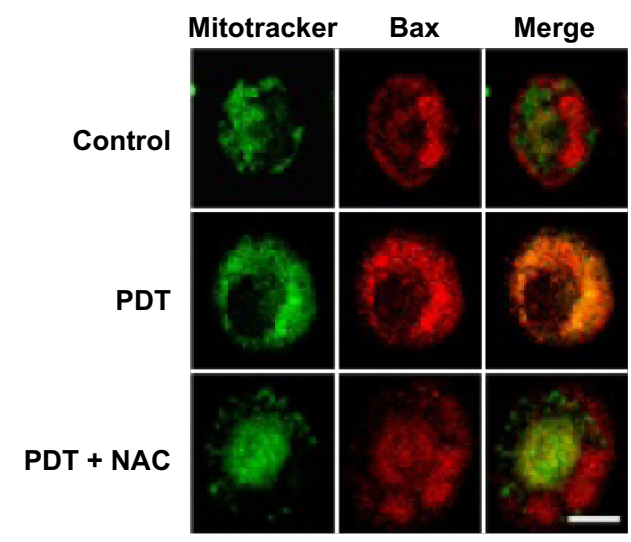

B

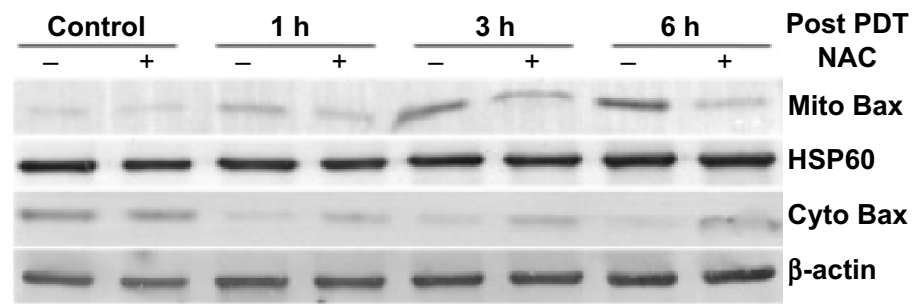

PDT + NAC
Mitotracker Cyto-C Merge

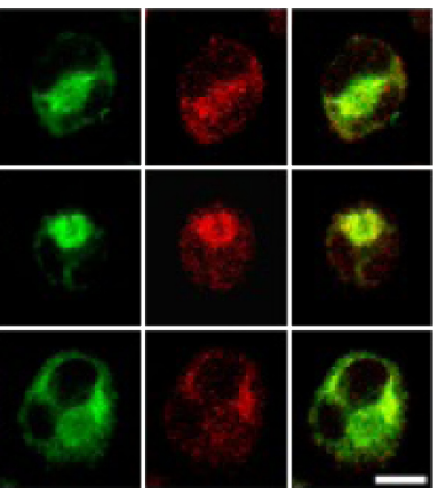

Mito Bax (NAC-) $\square$ Cyto Bax (NAC-)

Mito Bax (NAC+) 四 Cyto Bax (NAC+)

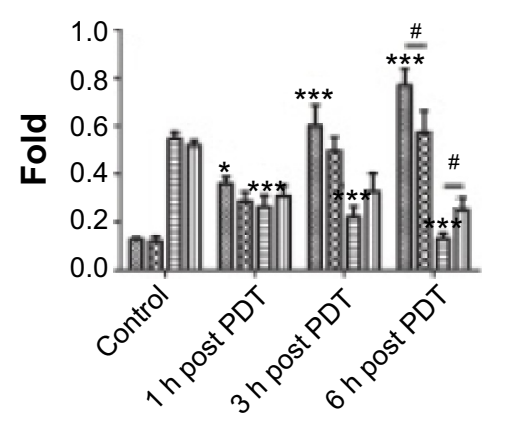

Mito cyto-C (NAC-) $\square$ Cyto cyto-C (NAC-)

Mito cyto-C (NAC+) $\square$ Cyto cyto-C (NAC+)

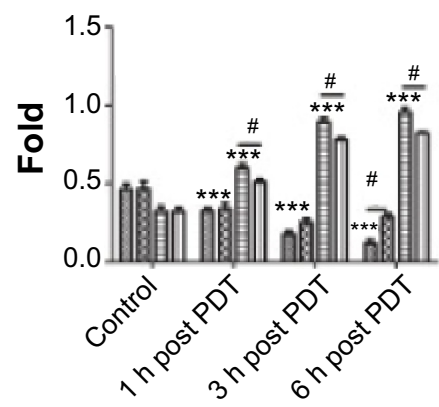

Figure 7 The blockade of Bax translocation and the release of cytochrome $C$ by NAC following PDT.

Notes: (A) Fluorescent photomicrograph of Bax and cytochrome C translocation blocked by NAC following PDT. (B) Blockade of Bax translocation by NAC following PDT. (C) Blockade of the release of cytochrome $\mathrm{C}$ by NAC following PDT. Mitochondrial protein levels were normalized to HSP60, and the cytosolic protein levels were normalized to $\beta$-actin. $* P<0.05$ vs control group; $* * * P<0.001$ vs control group; ${ }^{* P}<0.05$ vs PDT group. Scale bar $=0.01 \mathrm{~mm}$.

Abbreviations: PDT, photodynamic therapy; NAC, N-acetyl-L-cysteine; cyto-C, cytochrome C; h, hours.

use of NIR light in PDT may therefore represent a promising therapy in the noninvasive treatment of atherosclerotic vessels. We utilized the UCNP encapsulating photosensitizer Ce6 (UCNPs-Ce6) as an agent of NIR-PDT to investigate its effects on THP-1 macrophages in vitro.

Mesoporous silica materials (as ideal materials for hosting guest molecules of various sizes, shapes, and functionalities) have displayed great potential in the application of imaging, drug delivery, and cancer treatment. ${ }^{41-43}$
Compared with other types of hosts, such as PEGylated UCNPs and $\alpha$-CDlated UCNPs, the strongest advantage of mesoporous-silica-coated UCNPs is their ability to protect captured photosensitizers from extremely hostile environments, such as tumors characterized by different $\mathrm{pH}$ environments. ${ }^{17}$ Moreover, mesoporous-silica-coated UCNPs fit our strategy of selecting nanoparticles for use in the setting of AS. Recently, macrophage-targeted theranostic nanoparticles have been described. These nanoparticles 
may be activated by ROS in macrophage cells and have great potential in treating AS, but the advantages they offer remain unknown. ${ }^{44}$

The following characteristics were present after PDT treatment: presence of Hoechst 33258 and PI cell populations and TUNEL staining, and the activation of caspase-related proteins. We established that irradiated cells subjected to $16 \mu \mathrm{g} / \mathrm{mL}$ UCNPs-Ce6 experienced not only morphological changes but also DNA strand breakage (Figure 3). In contrast, apoptotic alterations were less evident in the control cells (the control, UCNPs-Ce6, and laser groups).

ROS are indispensable to the signal transduction pathways that regulate cell death and cellular redox states. However, the overproduction of ROS may damage lipids, proteins, and DNA. ${ }^{45}$ This study demonstrated that intracellular ROS production was increased significantly and peaked at 3 hours following PDT treatment (Figure 4B). There are two explanations for this increase in ROS: 1) the photosensitizer may transfer its energy to form highly reactive ${ }^{1} \mathrm{O}_{2}$ when oxygen is present in the environment as previous studies have reported that ${ }^{1} \mathrm{O}_{2}$ may be one of the mediators of the imbalance in intracellular ROS; ${ }^{29}$ and 2) the activated photosensitizer may react directly with organic substrates or via electron or hydrogen transfer to yield free radicals. ${ }^{28}$ Our results indicated that the ROS inhibitor, NAC, and the ${ }^{1} \mathrm{O}_{2}$ inhibitor, $\mathrm{NaN}_{3}$, significantly blocked intracellular ROS increases (Figure 4A). Furthermore, the scavenger of the hydroxyl radical, mannitol, also facilitated cell viability decreases following PDT. These results indicated that ${ }^{1} \mathrm{O}_{2}$ and ROS, particularly the hydroxyl radical, were the primary factors regulating the apoptosis induced by UCNPs-Ce6mediated PDT. However, it remains unknown whether ${ }^{1} \mathrm{O}_{2}$ triggers the increase in intracellular ROS. Furthermore, we determined the levels of MDA, which is the end product of lipid peroxidation, in the mitochondrial membrane following different treatments. The PDT group exhibited an elevated MDA level (Figure 4D), which may have been due to the much higher levels of ROS in the PDT group. The occurrence of lipid peroxidation secondary to oxidative stress may have altered the structure of the mitochondrial membrane, affected its physiological function, opened the MPTP, and resulted in apoptosis. ${ }^{46,47}$

An increase in ROS and a consequent loss of MMP were reported to be typical phenomena during mitochondriadependent apoptosis. ${ }^{48}$ Our study demonstrated that MMP diminished in a time-dependent manner, which was not consistent with the ROS-induced alterations following PDT. MMP diminished gradually within the first 2 hours before suddenly decreasing to a low level (Figure 5C). The first 2 hours following PDT was characterized by the accumulation of ROS in the cells, which affected the MMP only to a limited extent. However, the ROS peaked at 3 hours following PDT when the greatest damage to the mitochondria occurred, which may explain why the MMP deteriorated rapidly after 3 hours. Interestingly, the alterations in the MPTP during 5 hours following PDT treatment were characterized by different patterns of mitochondrial depolarization and ROS accumulation (Figure 6C). The alterations of the MPTP triggered a gradual decrease within the first 2 hours, which was consistent with the changes in mitochondrial depolarization, but it decreased rapidly during the following hour, which coincided with the maximum accumulation of ROS. The period between 3 and 5 hours was characterized by a slower rate of decline, which may also have been the result of ROS accumulation, as the level of intracellular ROS declined during that period. Comparisons of the two alteration patterns during the final 2 hours demonstrated that MMP decreased more markedly than MPTP, which was most likely because the opening of the MPTP favors mitochondrial depolarization. ${ }^{49}$ Furthermore, we observed that CYPD, rather than adenine nucleotide translocase and VDAC, regulated the opening of the MPTP following PDT treatment (Figure 6A, B). Coincidentally, CYPD also controlled the change in MMP (Figure 5B), which not only demonstrated that MPTP opening favors mitochondrial depolarization but also indicated that the MPTP subunit, CYPD, plays a vital role in regulating MMP loss. We concluded that the bursts of ROS indirectly triggered MMP alterations by influencing the MPTP.

The remarkable feature of mitochondrial-dependent apoptosis is the release of cytochrome $\mathrm{C}$ from mitochondria into cytosol. This process involves an interaction with apoptotic protease-activating factor-1 via caspase recruitment domains and the subsequent formation of an apoptosome, thus activating both caspase- 9 and caspase-3, which leads to PARP cleavage and initiation of the caspase cascade, ultimately resulting in apoptosis. ${ }^{50,51}$ Our results were in agreement with those from previous studies. We also observed that the translocation of Bax and cytochrome $\mathrm{C}$ were blocked by NAC following PDT treatment (Figure 7), which might be because ROS bursts following PDT play an important role in regulating the translocation and cytochrome $\mathrm{C} .{ }^{52}$ Our findings demonstrated that UCNPs-Ce6-mediated PDT induces apoptosis in THP-1 macrophages via the mitochondrial caspase pathway, which is activated by ROS bursts following PDT treatment (Figure 8). 


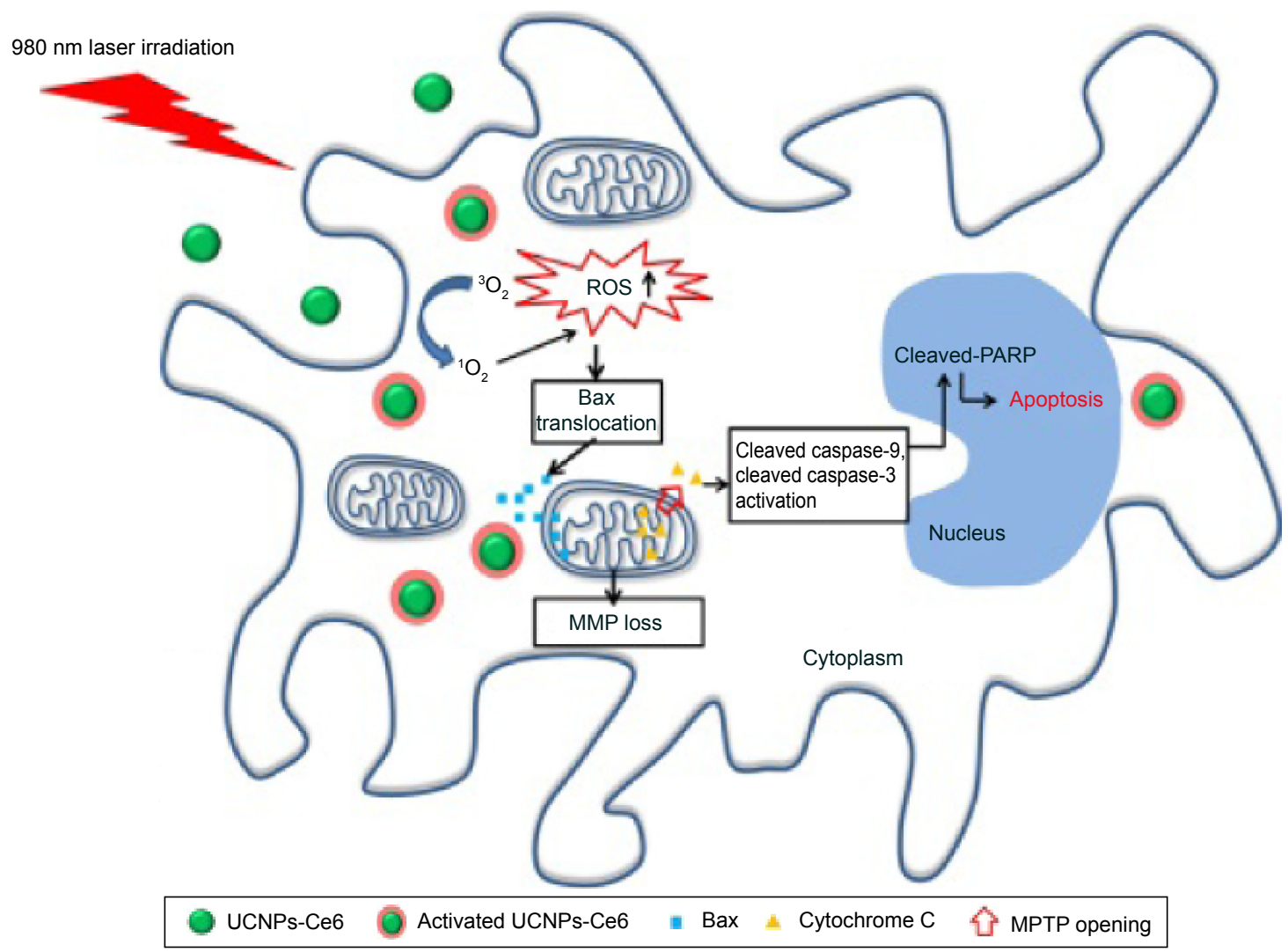

Figure 8 A schematic illustration of UCNPs-Ce6-mediated PDT and the mechanism of apoptosis in vitro.

Notes: Activated UCNPs-Ce6 in THP-I macrophages generates ' $\mathrm{O}_{2}$, resulting in the bursts of ROS. UCNPs-Ce6-mediated PDT results in apoptotic cell death most likely via mitochondrial-dependent death pathways triggered by ROS. ROS upregulation facilitates Bax translocation to the mitochondria as well as the release of cytochrome $\mathrm{C}$ into the cytoplasm via the MPTP, which causes the loss of MMP. Cytochrome $C$ in the cytoplasm activates several caspase-related apoptotic proteins, resulting in apoptosis.

Abbreviations: ROS, reactive oxygen species; PARP, poly(ADP-ribose) polymerase; MMP, mitochondrial membrane potential; UCNPs, upconversion nanoparticles; Ce6, chlorin e6; MPTP, mitochondrial permeability transition pore; ' $\mathrm{O}_{2}$, singlet oxygen; ${ }^{3} \mathrm{O}_{2}$, triplet oxygen.

In summary, our study established nanoparticle platforms as potential agents to induce macrophage apoptosis to attenuate the progression of AS. Our findings also contributed to the understanding of the complex cell death pathways induced by PDT and provided improvements to PDT in the setting of AS. However, given the complicated role played by macrophages in AS, additional studies involving animal AS models should be performed. Furthermore, the use of nanoparticles may be an interesting strategy for the treatment of inflammatory diseases.

\section{Conclusion}

UCNPs-Ce6-mediated PDT exhibited synergistic damage and apoptosis of THP-1 macrophages in vitro via the targeting of mitochondria, bursts of ROS, and translocation of Bax and cytochrome $\mathrm{C}$ to regulate the MPTP opening, thereby causing MMP depolarization and activation of mitochondrial caspase pathway. Our results imply that UCNPs-Ce6-mediated PDT, which realized not only the deep penetration depth but also the strategy of reducing the infiltration of macrophages in atherosclerotic plaque, might be a good treatment of AS.

\section{Acknowledgments}

This study was supported by the National Natural Science Foundation of China (81000688, 81271734, 81300200), the Heilongjiang Provincial Youth Science Foundation (QC2011C002), the Foundation of Science and Technology Innovation Talent of Harbin Science and Technology Bureau (2011RFQXS072), the Wu Liande Youth Science Foundation of Harbin Medical University (WLD-QN1104), the Postdoctoral Science-research Developmental Foundation of Heilongjiang Province (LBHQ12049), the China Postdoctoral Science Foundation funded project (20090460911), the Ministry of Education and the Research Foundation of the Health Department of Heilongjiang Province (2009-217).

\section{Disclosure}

The authors report no conflicts of interest in this work. 


\section{References}

1. Wang FP, Gao QP, Guo SY, et al. The sonodynamic effect of curcumin on THP-1 cell-derived macrophages. Biomed Res Int. 2013; 2013:737264.

2. Capewell S, Lloyd-Jones DM. Optimal cardiovascular prevention strategies for the 21st century. JAMA. 2010;304:2057-2058.

3. Moore KJ, Tabas I. Macrophages in the pathogenesis of atherosclerosis. Cell. 2011;145(3):341-355.

4. Yang EH, Gumina RJ, Lennon RJ, et al. Emergency coronary artery bypass surgery for percutaneous coronary interventions: changes in the incidence, clinical characteristics, and indications from 1979 to 2003. J Am Coll Cardiol. 2005;46(11):2004-2009.

5. Dolmans DE, Fukumura D, Jain RK. Photodynamic therapy for cancer. Nat Rev Cancer. 2003;3(5):380-387.

6. Castano AP, Mroz P, Hamblin MR. Photodynamic therapy and antitumour immunity. Nat Rev Cancer. 2006;6(7):535-545.

7. Tian G, Ren W, Yan L, et al. Red-emitting upconverting nanoparticles for photodynamic therapy in cancer cells under near-infrared excitation. Small. 2013;9(11):1929-1938.

8. Tang G, Hyman S, Schneider JH Jr, et al. Application of photodynamic therapy to the treatment of atherosclerotic plaques. Neurosurgery. 1993; 32(3):438-443.

9. Waksman R, McEwan PE, Moore TI, et al. PhotoPoint photodynamic therapy promotes stabilization of atherosclerotic plaques and inhibits plaque progression. J Am Coll Cardiol. 2008;52(12):1024-1032.

10. Peng $\mathrm{C}, \mathrm{Li} \mathrm{Y}$, Liang $\mathrm{H}$, et al. Detection and photodynamic therapy of inflamed atherosclerotic plaques in the carotid artery of rabbits. J Photochem Photobiol B. 2011;102(1):26-31.

11. Liu Q, Sun Y, Li C, et al. 18F-labeled magnetic-upconversion nanophosphors via rare-earth cation-assisted ligand assembly. ACS Nano. 2011;5(4):3146-3157.

12. Cheng L, Yang K, Li Y, et al. Facile preparation of multifunctional upconversion nanoprobes for multimodal imaging and dual-targeted photothermal therapy. Angew Chem Int Ed Engl. 2011;50(32):7385-7390.

13. Frangioni JV. In vivo near-infrared fluorescence imaging. Curr Opin Chem Biol. 2003;7(5):626-634.

14. Chatterjee DK, Rufaihah AJ, Zhang Y. Upconversion fluorescence imaging of cells and small animals using lanthanide doped nanocrystals. Biomaterials. 2008;29(7):937-943.

15. Cao Z, Zhu W, Wang W, et al. Stable cerasomes for simultaneous drug delivery and magnetic resonance imaging. Int $J$ Nanomedicine. 2014;9:5103.

16. Qian HS, Guo HC, Ho PC, et al. Mesoporous-Silica-coated upconversion fluorescent nanoparticles for photodynamic therapy. Small. 2009;5(20):2285-2290.

17. Guo H, Qian H, Idris NM, Zhang Y. Singlet oxygen-induced apoptosis of cancer cells using upconversion fluorescent nanoparticles as a carrier of photosensitizer. Nanomedicine. 2010;6(3):486-495.

18. Xiong L, Chen Z, Tian Q, Cao T, Xu C, Li F. High contrast upconversion luminescence targeted imaging in vivo using peptide-labeled nanophosphors. Anal Chem. 2009;81(21):8687-8694.

19. Cheng L, Yang K, Shao M, Lee ST, Liu Z. Multicolor in vivo imaging of upconversion nanoparticles with emissions tuned by luminescence resonance energy transfer. $J$ Phys Chem C. 2011;115(6): 2686-2692.

20. Wang C, Cheng L, Liu Z. Drug delivery with upconversion nanoparticles for multi-functional targeted cancer cell imaging and therapy. Biomaterials. 2011;32(4):1110-1120.

21. Chou LC, Yang JS, Huang LJ, et al. The synthesized 2-(2fluorophenyl)-6, 7-methylenedioxyquinolin-4-one (CHM-1) promoted $\mathrm{G} 2 / \mathrm{M}$ arrest through inhibition of CDK1 and induced apoptosis through the mitochondrial-dependent pathway in CT-26 murine colorectal adenocarcinoma cells. J Gastroenterol. 2009;44(10):1055-1063.

22. Wang C, Tao H, Cheng L, Liu Z. Near-infrared light induced in vivo photodynamic therapy of cancer based on upconversion nanoparticles. Biomaterials. 2011;32(26):6145-6154.
23. Tabas I. Macrophage death and defective inflammation resolution in atherosclerosis. Nat Rev Immunol. 2010;10(1):36-46.

24. Martinet W, Verheye S, De Meyer GR. Selective depletion of macrophages in atherosclerotic plaques via macrophage-specific initiation of cell death. Trends Cardiovasc Med. 2007;17(2):69-75.

25. Copley L, van der Watt P, Wirtz KW, Parker MI, Leaner VD. Photolon $^{\mathrm{TM}}$, a chlorin e6 derivative, triggers ROS production and lightdependent cell death via necrosis. Int J Biochem Cell Biol. 2008; 40(2):227-235.

26. Ong CY, Ling SK, Ali RM, et al. Systematic analysis of in vitro photo-cytotoxic activity in extracts from terrestrial plants in Peninsula Malaysia for photodynamic therapy. J Photochem Photobiol B. 2009;96(3):216-222.

27. Liu L, Zhang Z, Xing D. Cell death via mitochondrial apoptotic pathway due to activation of Bax by lysosomal photodamage. Free Radic Biol Med. 2011;51(1):53-68.

28. Ricchelli F, Šileikyte J, Bernardi P. Shedding light on the mitochondrial permeability transition. Biochim Biophys Acta. 2011;1807(5): 482-490.

29. Magi B, Ettorre A, Liberatori S, et al. Selectivity of protein carbonylation in the apoptotic response to oxidative stress associated with photodynamic therapy: a cell biochemical and proteomic investigation. Cell Death Differ. 2004;11(8):842-852.

30. Song W, Cui H, Zhang R, et al. Apoptosis of SAS cells induced by sonodynamic therapy using 5 -aminolevulinic acid sonosensitizer. Anticancer Res. 2011;31(1):39-45.

31. Duchen MR. Contributions of mitochondria to animal physiology: from homeostatic sensor to calcium signalling and cell death. J Physiol. 1999;516(1):1-17.

32. Zamzami N, Kroemer G. The mitochondrion in apoptosis: how Pandora's box opens. Nat Rev Mol Cell Biol. 2001;2(1):67-71.

33. McBride HM, Neuspiel M, Wasiak S. Mitochondria: more than just a powerhouse. Curr Biol. 2006;16(14):R551-R560.

34. Smith DJ, Ng H, Kluck RM, et al. The mitochondrial gateway to cell death. IUBMB Life. 2008;60(6):383-389.

35. Hung HI, Schwartz JM, Maldonado EN, et al. Mitoferrin-2-dependent mitochondrial iron uptake sensitizes human head and neck squamous carcinoma cells to photodynamic therapy. J Biol Chem. 2013; 288(1):677-686

36. Woodfield K, Ruck A, Brdiczka D, et al. Direct demonstration of a specific interaction between cyclophilin-D and the adenine nucleotide translocase confirms their role in the mitochondrial permeability transition. Biochem J. 1998;336:287-290.

37. Miura T, Tanno M. The mPTP and its regulatory proteins: final common targets of signalling pathways for protection against necrosis. Cardiovasc Res. 2012;94:181-189.

38. Youle RJ, Strasser A. The BCL-2 protein family: opposing activities that mediate cell death. Nat Rev Mol Cell Biol. 2008;9(1): $47-59$.

39. Buytaert E, Callewaert G, Hendrickx N, et al. Role of endoplasmic reticulum depletion and multidomain proapoptotic BAX and BAK proteins in shaping cell death after hypericin-mediated photodynamic therapy. FASEB J. 2006;20(6):756-758.

40. Lloyd-Jones D, Adams RJ, Brown TM, et al. Heart disease and stroke statistics - 2010 update a report from the American Heart Association. Circulation. 2010;121(7):e46-e215.

41. Lin G, Wang X, Yin F, et al. Passive tumor targeting and imaging by using mercaptosuccinic acid-coated near-infrared quantum dots. Int J Nanomed. 2015;10:335.

42. Lee JY, Kim JS, Cho HJ, et al. Poly (styrene)-b-poly (DL-lactide) copolymer-based nanoparticles for anticancer drug delivery. Int J Nanomed. 2014;9:2803.

43. Arora S, Swaminathan SK, Kirtane A, et al. Synthesis, characterization, and evaluation of poly (D, L-lactide-co-glycolide)-based nanoformulation of miRNA-150: potential implications for pancreatic cancer therapy. Int J Nanomed. 2014;9:2933. 
44. Kim H, Kim Y, Kim IH, et al. ROS-responsive activatable photosensitizing agent for imaging and photodynamic therapy of activated macrophages. Theranostics. 2014;4(1):1.

45. Kang SU, Cho JH, Chang JW, et al. Nonthermal plasma induces head and neck cancer cell death: the potential involvement of mitogenactivated protein kinase-dependent mitochondrial reactive oxygen species. Cell Death Dis. 2014;5(2):e1056.

46. Jin H, Zhong X, Wang Z, et al. Sonodynamic effects of hematoporphyrin monomethyl ether on CNE-2 cells detected by atomic force microscopy. $J$ Cell Biochem. 2011;112(1):169-178.

47. Li JH, Yue W, Huang Z, et al. Calcium overload induces C6 rat glioma cell apoptosis in sonodynamic therapy. Int J Radiat Biol. 2011; 87(10):1061-1066.

48. Vaux DL, Korsmeyer SJ. Cell death in development. Cell. 1999;96(2): $245-254$.
49. Petronilli V, Penzo D, Scorrano L, et al. The mitochondrial permeability transition, release of cytochrome $\mathrm{c}$ and cell death correlation with the duration of pore openings in situ. J Biol Chem. 2001; 276(15):12030-12034.

50. Van Loo G, Saelens X, Van Gurp M, et al. The role of mitochondrial factors in apoptosis: a Russian roulette with more than one bullet. Cell Death Differ. 2002;9(10):1031-1042.

51. Li X, Zheng L, Kou J, et al. The efficacy and mechanism of apoptosis induction by hypericin-mediated sonodynamic therapy in THP-1 macrophages. Int J Nanomed. 2015;10:821-838.

52. Yoo JO, Lim YC, Kim YM, et al. Transglutaminase 2 promotes both caspase-dependent and caspase-independent apoptotic cell death via the calpain/Bax protein signaling pathway. J Biol Chem. 2012; 287(18):14377-14388.
International Journal of Nanomedicine

\section{Publish your work in this journal}

The International Journal of Nanomedicine is an international, peerreviewed journal focusing on the application of nanotechnology in diagnostics, therapeutics, and drug delivery systems throughout the biomedical field. This journal is indexed on PubMed Central, MedLine, CAS, SciSearch ${ }^{\circledR}$, Current Contents ${ }^{\circledR} /$ Clinical Medicine,

\section{Dovepress}

Journal Citation Reports/Science Edition, EMBase, Scopus and the Elsevier Bibliographic databases. The manuscript management system is completely online and includes a very quick and fair peer-review system, which is all easy to use. Visit http://www.dovepress.com/ testimonials.php to read real quotes from published authors. 